This item was submitted to Loughborough's Research Repository by the author.

Items in Figshare are protected by copyright, with all rights reserved, unless otherwise indicated.

\title{
Damping of flexural vibrations in rectangular plates using the acoustic black
} hole effect

PLEASE CITE THE PUBLISHED VERSION

http://dx.doi.org/10.1016/j.jsv.2010.05.019

PUBLISHER

(C) Elsevier Ltd.

VERSION

SMUR (Submitted Manuscript Under Review)

LICENCE

CC BY-NC-ND 4.0

REPOSITORY RECORD

O'Boy, D.J., Victor V. Krylov, and V. Kralovic. 2012. "Damping of Flexural Vibrations in Rectangular Plates Using the Acoustic Black Hole Effect”. figshare. https://hdl.handle.net/2134/9958. 
This item was submitted to Loughborough's Institutional Repository (https://dspace.lboro.ac.uk/) by the author and is made available under the following Creative Commons Licence conditions.

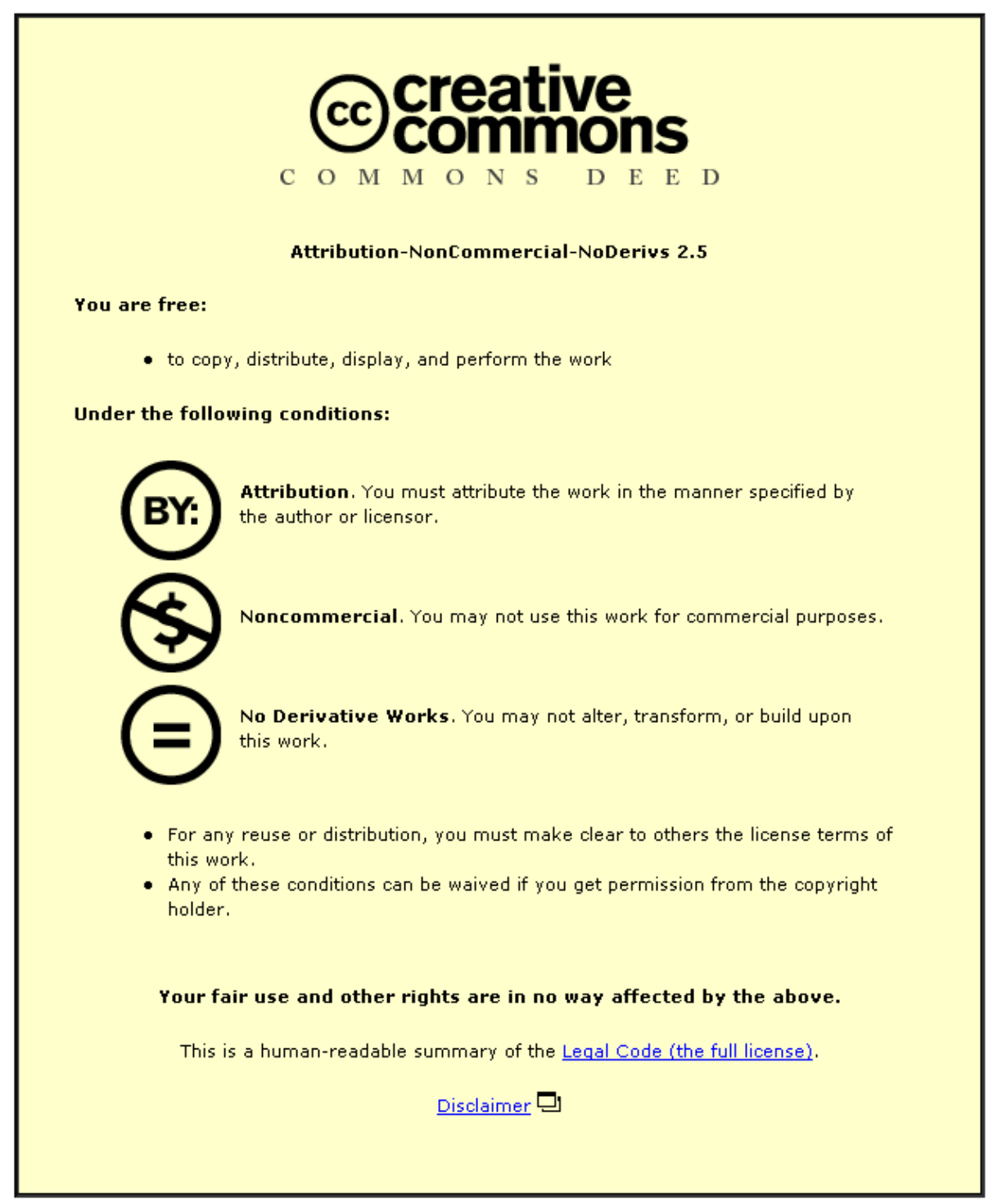

For the full text of this licence, please go to: http://creativecommons.org/licenses/by-nc-nd/2.5/ 


\title{
Damping of flexural vibrations in rectangular plates using the acoustic black hole effect
}

\author{
D. J. O'Boy*, V. V. Krylov V. Kralovic \\ Department of Aeronautical and Automotive Engineering, Loughborough \\ University, Leicestershire, LE11 3TU, UK
}

\begin{abstract}
The reduction of flexural vibration in plate structures has been investigated using the recently reported acoustic black hole effect for flexural wave reflection in plates with the local thickness varying according to $h(x)=\epsilon x^{m}$ and $m \geq 2$. Since sharp edges of such plates (wedges) are always truncated before $x=0$, the real reflection coefficients are relatively high, therefore the application of a small amount of damping is required to achieve large reductions in vibration amplitude. This paper presents a numerical model of a plate incorporating an acoustic black hole wedge, with predictions for vibration amplitudes. These are compared to equivalent experimental measurements for a range of applied damping layers. It is concluded that the above-mentioned power-law wedges can be used as effective vibration dampers in plate structures over a wide frequency range of interest.
\end{abstract}

Key words: Acoustic black hole effect, Vibration damping, Rectangular plates, Numerical model

\section{Introduction}

The efficient damping of flexural plate vibrations in different structures continues to pose challenges for various branches of engineering. Traditionally, vibration attenuation has been achieved by utilising thick damping layers spread over the whole structural surface [1][2][3]. However, for many applications, it is not possible or desirable to incorporate this damping material without impacting other design factors or constraints. For these applications,

\footnotetext{
* Corresponding author.

Email address: d.j.oboy@lboro.ac.uk (D. J. O'Boy).

URL: (D. J. O'Boy).
} 
other available methods of damping resonant vibrations, such as construction of gradual impedance interfaces to reduce edge reflections of flexural waves, can be considered [4].

A different approach to damping resonant flexural vibrations via reduction of edge reflections has been proposed by Mironov [5]. He has pointed out that a flexural wave propagating towards a sharp edge of a quadratically tapered plate (wedge) slows down in such a way that it requires an indefinite time to reach the edge, so that even a negligibly small material attenuation can cause all of the wave energy to be absorbed near the edge, with no energy being reflected. Such ideal quadratic wedges thus behave almost like acoustic black holes, where the term "acoustic black hole effect" has been used to represent a modification which can be made to one or more of the plate boundaries such that energy can travel into the wedge but not travel out. The flexural wave travels into the wedge where the extensional and compressive movements cause conversion of the kinetic energy to heat, due to an inherent loss factor present in the material. This effective rate of dissipation is amplified by the tapered geometry.

Although the analogy can apply to any vibration system involving damping mechanisms of this kind (for example gradual impedance changes in the materials), it is used here to refer to the topic of modifying plate structures with damped wedges or inclusions of power-law profile.

Unfortunately, because of the deviations of real manufactured wedges from the ideal power-law shapes, largely due to ever-present truncations of the wedge edges, the reflection coefficients in real quadratic wedges remain as high as 50-70 percent, so that they can not be used as practical vibration dampers.

An important step to make the above mentioned acoustic black holes work practically has been made by Krylov [6] (see also [7][8]) who has suggested covering wedge surfaces near sharp edges by thin strips of damping layers, for example polymeric films. Note that the idea of applying absorbing layers for damping flexural vibrations in plates of constant thickness has been used successfully since 1950 [3]. The new aspect of this idea, which is used in the proposed approach, is to apply such absorbing layers to plates of variable thickness (or wedges) characterised by the specific power-law geometry to achieve maximum damping.

According to the theoretical calculations [6][7][8], wedges of power-law profile covered by thin absorbing layers can be very efficient damping systems, with flexural wave reflection coefficients as low as 1-3 percent. In the recent experimental work [9], thin layers of damping material have been applied to a wedge of power-law profile, with sharp reductions in resonant peaks being observed. Although a detailed comparison of the theory with experimental 
measurements was not carried out in this work, the conclusion drawn was that power-law profile wedges with small amounts of damping material can be efficient practical vibration dampers, despite the truncations.

The present paper describes the results of further theoretical and experimental research into the practical use of acoustic black hole dampers of flexural vibrations. The aim of this paper is to investigate the efficiency of application of different power-law wedge attachments to damping resonant vibrations in a rectangular plate considered as an example of a practical basic structure. A full comparison will be provided between the frequency response functions of a simple rectangular plate with and without the addition of a wedge attachment shaped to fit the profile of an acoustic black hole, as illustrated in Fig. 1. The paper also provides a comparison between a numerical model of plate vibration and experimental measurements.

The frequency response functions for the different plates will be compared by examination of the peak mobility amplitude at certain frequencies. For example, given a resonant peak at $1 \mathrm{kHz}$, the peak amplitude of the different test plates are compared. A summary table is also provided, which analyses the amplitude over a wide frequency range, to mitigate against the chances of selecting a measurement point located on a vibration anti-node.

The numerical bending plate model is described in section 2. It provides the vibration response of the plate structure in question undergoing harmonic excitation. One edge of the plate may incorporate either a free edge, or a wedge profile, allowing direct predictions to be made of a plate with and without an acoustic black hole profile.

Section 3 contains a description of the plate structure manufacturing process and the experimental testing. In particular, the experimental rig is described in section 3.1. A comparison of the driving and cross point mobility responses for a simple rectangular plate is given in section 3.2 to validate the numerical model by the experimental results.

In section 3.3, the simple rectangular plate is compared with a similar plate with a wedge profile, followed by a comparison of the wedge response with and without damping tape applied (section 3.4). Finally, the obtained results are discussed and conclusions presented in section 4 .

\section{Numerical bending plate model}

In order to evaluate the performance of several rectangular plates incorporating wedges of power-law profile, a numerical model has been developed. This 
section contains a derivation of a numerical model using a simplified bending plate model which yields the velocity of the plate normal to the surface $\dot{w} \mathrm{~ms}^{-1}$ when a harmonic force $p_{0} \mathrm{~N}$ is applied.

The aim of the numerical model is to determine the driving point mobility response of the plate with attached wedges (the velocity normal to the plate surface coincident with the forcing location) and the cross point mobility (where the response point is away from the forcing).

The cross point mobility response is shown in Fig. 2, where the forcing point is located at $\left(x_{0}, y_{0}\right) \mathrm{m}$ and the measurement point $(x, y) \mathrm{m}$ of a plate with basic dimensions $(x=b-a, y=2 c) \mathrm{m}$. The numerical model consists of two constant thickness plates, where a shear force $p_{0}$ can be applied at the join $x=x_{0}$ as a boundary condition. This avoids the requirement to consider the loading on the plate in the immediate equation of motion.

At one end of the plate, $x=a$, a test section is located, which can either be another constant thickness plate, a free end or a wedge with a specified profile which terminates at a truncation point $x=x_{t}$. It will be considered that all other edges in $y= \pm c$ are simply supported, while the surface at $x=b$ is free.

The numerical model includes both the representation of the constant thickness plate, the wedge and a damping layer which can be applied to the plate surface. The material properties of the wedge will use the subscript $w$ and the damping layer $D$.

A classical bending plate equation of motion for a constant thickness plate was used [10],

$$
\left(\nabla^{2}\right)\left(-D \nabla^{2}\right) w(x, y, t)=\rho h \frac{\partial^{2} w(x, y, t)}{\partial t^{2}},
$$

where $D \mathrm{Nm}$ is the bending stiffness, $w(x, y, t) \mathrm{m}$ is the plate normal displacement, $h \mathrm{~m}$ is the constant thickness and the operator $\nabla^{4}=\left(\partial^{2} / \partial x^{2}+\partial^{2} / \partial y^{2}\right)^{2}$. The density of the plate is $\rho \mathrm{kgm}^{-3}$.

As the constant thickness plate is composed of two different smaller plates, the solution is presumed to be linear with small amplitude in the form of a Fourier sum in the dimensions $x, y$ and the time $t$,

$$
w(x, y, t)=\left\{\begin{array}{l}
\sum A_{j}^{-} e^{\left(k_{j}\left[x-x_{0}\right]\right)} e^{\left(\mathrm{i} k_{y}\left[y-y_{0}\right]\right)} e^{-\mathrm{i} \omega t} \text { for } x \leq x_{0}, \\
\sum A_{j}^{+} e^{\left(k_{j}\left[x-x_{0}\right]\right)} e^{\left(\mathrm{i} k_{y}\left[y-y_{0}\right]\right)} e^{-\mathrm{i} \omega t} \text { for } x>x_{0}
\end{array}\right.
$$

where $k_{j}, k_{y}$ are the wavenumbers in the $x$ and $y$ directions respectively and $A_{j}$ are the Fourier coefficients to be determined. The displacement for $x>x_{0}$ 
will be denoted $w^{+}$and for $x \leq x_{0}$ will be $w^{-}$. The frequency $\omega=2 \pi f$, where $f$ is the excitation frequency in Hz.

At this point, the boundary conditions applied to the plate edges $y= \pm c$ are simply supported at all times, so that zero bending moment and displacement exist. Then, the only applicable values of the wavenumber $k_{y}=n \pi / 2 c$ where the mode number $n=1,2 \ldots$ for odd and even modes of vibration [10]. The solutions in $y$ are then comprised of a sum of the solutions for each wavenumber, $w\left(y, k_{j}, \omega\right)=\sum_{n=1}^{n_{\max }} w\left(k_{j}, \omega\right) e^{\left(\mathrm{i} k_{y}\left[y-y_{0}\right]\right)}$.

For the solutions in the dimension $x$, substitution of Eq. (2) into the equation of motion, Eq. (1), leads to a bi-quadratic equation in $k_{j}$ (therefore $j=1,2,3,4$.),

$$
\left(D k_{j}^{4}+\left(-2 D k_{y}^{2}\right) k_{j}^{2}+\left(D k_{y}^{4}-\rho h \omega^{2}\right)\right) w(x, y, t)=0 .
$$

For any given mode of vibration in $y$ and assuming no trivial solution, there are four roots of $k_{j}$ in Eq. (3) and four amplitudes to determine in Eq. (2) for each of the smaller plates. These roots are calculated numerically for every wavenumber $k_{y}$ and frequency $\omega$. For the case where $k_{y}=0$, these roots are two propagating and two evanescent waves,

$k=\left[\left(\omega^{2} \rho h / D\right)^{\frac{1}{4}},-\left(\omega^{2} \rho h / D\right)^{\frac{1}{4}}, \quad \mathrm{i}\left(\omega^{2} \rho h / D\right)^{\frac{1}{4}}, \quad-\mathrm{i}\left(\omega^{2} \rho h / D\right)^{\frac{1}{4}}\right]$.

The displacement of the plate is given as a Fourier sum, with the Fourier coefficients for the plate $x \leq x_{0}$ given as $A_{j}^{-}$and the Fourier coefficients for positions $x>x_{0}$ as $A_{j}^{+}$.

\subsection{Boundary conditions for a simple rectangular plate}

To find the eight Fourier coefficients for the simple rectangular plate requires eight boundary conditions. The first two specify the connection between the composite plate and the test section (which could be a damped plate, wedge or power-law profile section). If this is a free edge, the conditions shown are bending moment and shear force (the following equations do not exactly equal the bending moment or shear force as the stiffness terms are omitted as they cancel on all sides, however they are the kinematic quantities which lead to them). Otherwise the geometry is matched on both sides, as follows.

Boundary condition 1, 2: At $x=a$, specify plate second and third derivative.

$$
\begin{aligned}
& \frac{\partial^{2} w^{-}(x, \omega)}{\partial x^{2}} \text { at } x=a . \\
& \frac{\partial^{3} w^{-}(x, \omega)}{\partial x^{3}} \text { at } x=a .
\end{aligned}
$$


At the far end of the composite plate, it is assumed that the edges are free to move without restriction.

Boundary condition 3, 4: At $x=b$, specify plate bending moment and shear force.

$$
\begin{gathered}
\frac{\partial^{2} w^{+}(x, \omega)}{\partial x^{2}}-\nu k_{y}^{2} w^{+}(x, \omega)=0 \text { at } x=b . \\
\frac{\partial^{3} w^{+}(x, \omega)}{\partial x^{3}}-(2-\nu) k_{y}^{2} \frac{\partial w^{+}(x, \omega)}{\partial x}=0 \text { at } x=b .
\end{gathered}
$$

At the join between the two plates $x=x_{0}$, continuity of displacement, slope, second and third derivatives are applied. At this point the Fourier transform of the forcing load is introduced as a delta force, normalised by the bending stiffness $p_{0}(\omega)=\delta / D$.

Boundary condition 5-8: At $x=x_{0}$, specify continuity of displacement, slope, second and third derivative.

$$
\begin{gathered}
w^{+}(x, \omega)-w^{-}(x, \omega)=0 \text { at } x=x_{0} . \\
\frac{\partial w^{+}(x, \omega)}{\partial x}-\frac{\partial w^{-}(x, \omega)}{\partial x}=0 \text { at } x=x_{0} . \\
\frac{\partial^{2} w^{+}(x, \omega)}{\partial x^{2}}-\frac{\partial^{2} w^{-}(x, \omega)}{\partial x^{2}}=0 \text { at } x=x_{0} . \\
\frac{\partial^{3} w^{+}(x, \omega)}{\partial x^{3}}-\frac{\partial^{3} w^{-}(x, \omega)}{\partial x^{3}}=\delta / D \text { at } x=x_{0} .
\end{gathered}
$$

\subsection{Numerical solution method}

With eight boundary conditions and eight unknowns, the Fourier coefficients are determined using a simple matrix operation. The unknown Fourier coefficients are placed in a matrix $\mathbf{A}$, which is eight by one in size $\left(\mathbf{A}=\left[A_{j}^{-}, A_{j}^{+}\right]^{T}\right)$. The right hand side of the boundary conditions are placed in a matrix $\mathbf{W}$, which is also eight by one (only the final entry is non-zero). The Fourier transformed boundary conditions are placed in an eight by eight matrix $\mathbf{X}$, where $\mathbf{W}=\mathbf{X A}$.

This matrix operation is solved numerically in Matlab, by substituting the material properties of the plate into Eq. (3) and determining the roots $k_{j}$. The matrix $\mathbf{X}$ is filled and inverted, which can be used to find the Fourier amplitudes $\mathbf{A}$ and by using Eq. (2), the displacement of the plate.

This simple numerical method is applied over a sum in $k_{y}$ to a limit $n_{\max }=30$ and frequencies $\omega$ between 0 and $10 \mathrm{kHz}$. The point mobility is obtained from the displacement response using $\dot{w}(\omega) / p_{0}=\mathrm{i} \omega w(\omega) / p_{0}$ where the dot represents a differentiation with respect to time. 
The numerical model was initialised with a plate of dimensions $a=0.05 \mathrm{~m}, b=$ $0.330 \mathrm{~m}, c=0.0975 \mathrm{~m}$ and a thickness of $h=5.04 \mathrm{~mm}$. The material properties of the plate are a Young's modulus $E=190 \mathrm{GPa}$, density $\rho=7850 \mathrm{kgm}^{-3}$ and Poisson's ratio of 0.3. A small damping coefficient was incorporated into the Young's modulus to represent the very low damping of cold rolled mild steel, $\eta=0.6 \%$ (as discussed in section 2.5)[3].

With reference to Fig. 2, the coordinates of the harmonic forcing load are $\left(x_{0}, y_{0}\right)=(0.19,0.00) \mathrm{m}$, with the measurement point of the acceleration given by $(x, y)=(0.237,0.033) \mathrm{m}$ (the forcing is always located along the centre-line of the plate). All results are generated with a frequency sampling interval of $10 \mathrm{~Hz}$.

\subsection{Modifications to the boundary condition for the wedge attachment}

The previous section contains a method to determine the two dimensional plate displacement when all of the edges in $x$ are free. In this section, the equations will be developed which allow a two dimensional wedge of specified profile to be attached to one end of the plate. The theory in this section also utilises the classical bending plate equation of motion, with similar boundary conditions and can therefore be joined straight onto one edge of the constant thickness plate described in the previous section.

It is assumed that the wedge will follow a power-law profile with the local thickness of the wedge given by,

$$
h_{w}(x)=\epsilon x^{m},
$$

where $\epsilon$ is a positive constant. Ideally, the local thickness decreases to zero at $x=0$, however, a truncation point is introduced at $x=x_{t}$ where a real manufactured wedge ends with a vertical free edge. The thickness of the wedge thus increases to a maximum $h_{0}$ at $x=a$ where $\epsilon=h_{0} / a^{m}$. This enables it to match the constant thickness plate as shown in Fig. 1.

The derivation of the wave solutions present in a wedge profile follows that of Krylov and Tilman [7], however, oblique incidence of waves should be considered as well for the purpose of calculation of mobilities. To match the constant thickness plate, the wedge has a width of $2 c$ with identical boundary conditions on the sides.

As the thickness of the wedge decreases from $h_{0}$ to nearly zero, the derivation assumes that the change of cross sectional area with a change in $x$ is small enough that derivatives of order greater than two may be neglected. The solution will then be valid for all positions except at the very end of the 
wedge tip. The equation of motion for a wedge is then,

$$
\begin{aligned}
& \frac{\partial^{2}}{\partial x^{2}}\left[D_{w}\left(\frac{\partial^{2} w_{w}(x, y, t)}{\partial x^{2}}+\nu \frac{\partial^{2} w_{w}(x, y, t)}{\partial y^{2}}\right)\right]+2(1-\nu) \frac{\partial^{2}}{\partial x \partial y}\left[D_{w} \frac{\partial^{2} w_{w}(x, y, t)}{\partial x \partial y}\right] \\
& +\frac{\partial^{2}}{\partial y^{2}}\left[D_{w}\left(\frac{\partial^{2} w_{w}(x, y, t)}{\partial y^{2}}+\nu \frac{\partial^{2} w_{w}(x, y, t)}{\partial x^{2}}\right)\right]+\rho_{w} h_{w} \frac{\partial^{2} w_{w}(x, y, t)}{\partial t^{2}}=0
\end{aligned}
$$

where $D_{w}(x), \rho_{w}$ and $h_{w}(x)$ are the bending stiffness, density and thickness of the wedge respectively. The approximate (ray theory) solution for the displacement normal to the wedge surface can be sought in the form [11].

$$
w_{w}(x, y, t)=B(x) e^{\mathrm{i} k_{p} S(x)} e^{\mathrm{i} k_{y}\left[y-y_{0}\right]} e^{-\mathrm{i} \omega t},
$$

where $B(x)$ and $S(x)$ are slowly varying amplitude function and x-dependent part of the eiconal (phase) function, $S(x, y)=S(x)+k_{y} y$ respectively. The plate wavenumber $k_{p}$ is included at this stage as a constant which does not vary with $x$ or the plate thickness. It is used to non-dimensionalise the wavenumber in the wedge and can be related to the plate velocity $c_{p}$ using $\omega^{2}=k_{p}^{2} c_{p}^{2}$, where $c_{p}=\sqrt{E_{w} /\left(\rho_{w}\left(1-v_{w}^{2}\right)\right)}$.

Substitution of this solution (14) into the equation of motion, Eq. (13) and retention of higher order terms only yields the following equation for $S(x, y)$, or the so-called eiconal equation [11].

$$
|\nabla S(x, y)|^{4}=\frac{k_{a}(x)^{4}}{k_{p}^{4}}=n_{a}(x)^{4}
$$

where $k_{a}(x)=\left[\rho_{w} h_{w}(x) \omega^{2} / D_{w}(x)\right]^{1 / 4}$ and $n_{a}(x)$ is the corresponding refraction coefficient. Keeping in mind that $S(x, y)=S(x)+k_{y} y$, rewrite Eq. (15) as a fourth order equation in the first derivative of $S(x)$.

$$
\left(\frac{\partial S(x)}{\partial x}\right)^{4}+\left(\frac{\partial S(x)}{\partial x}\right)^{2}\left(\frac{2 k_{y}^{2}}{k_{p}^{2}}\right)+\left(\frac{k_{y}^{4}}{k_{p}^{4}}-\frac{\rho_{w} h_{w}(x) \omega^{2}}{D_{w}(x) k_{p}^{4}}\right)=0 .
$$

There are four roots to this equation, representing the propagating and evanescent waves in either the positive or negative $x$ directions. The height profile is now applied from Eq. (12) where $\gamma=\left(12^{1 / 2} k_{p} / \epsilon k_{y}^{2}\right)$.

$$
\frac{\partial S(x)}{\partial x}= \pm \frac{k_{y}}{k_{p}}\left(-1 \pm \gamma x^{-m}\right)^{1 / 2} .
$$

If $k_{y}=0$, the result from Ref [7] is obtained, where the first positive root is, $\partial S(x) / \partial x=(12)^{1 / 4}\left(\epsilon k_{p}\right)^{-1 / 2} x^{-m / 2}$. When $k_{y} \neq 0$, it is convenient to use the 
binomial expansion of Eq. (17) for $\left|x^{m} / \gamma\right|<1$. A binomial expansion provides the first terms of the series where the latter terms are important for the higher order modes in the $y$ direction of the plate (the oblique incidence waves which differentiate the 1D beam analysis provided in the literature from the $2 \mathrm{D}$ plate applied in this paper). Two solutions are available from the square root,

$$
\begin{aligned}
& \frac{\partial S_{1}(x)}{\partial x}=12^{\frac{1}{4}}\left(\epsilon k_{p}\right)^{-\frac{1}{2}} x^{-\frac{m}{2}}-\frac{1}{2} 12^{-\frac{1}{4}} k_{y}^{2} \epsilon^{\frac{1}{2}} k_{p}^{-\frac{3}{2}} x^{\frac{m}{2}}-\frac{1}{8} 12^{-\frac{3}{4}} k_{y}^{4} \epsilon^{\frac{3}{2}} k_{p}^{-\frac{5}{2}} x^{\frac{3 m}{2}} \\
& \frac{\partial S_{2}(x)}{\partial x}=\mathrm{i} 12^{\frac{1}{4}}\left(\epsilon k_{p}\right)^{-\frac{1}{2}} x^{-\frac{m}{2}}+\frac{\mathrm{i}}{2} 12^{-\frac{1}{4}} k_{y}^{2} \epsilon^{\frac{1}{2}} k_{p}^{-\frac{3}{2}} x^{\frac{m}{2}}-\frac{\mathrm{i}}{8} 12^{-\frac{3}{4}} k_{y}^{4} \epsilon^{\frac{3}{2}} k_{p}^{-\frac{5}{2}} x^{\frac{3 m}{2}}
\end{aligned}
$$

Integrating the term with respect to the position $x$, an expression is obtained for the wavenumber function $S(x)$. This set of equations is directly comparable to Krylov and Tilman [7] who finds that it is of the form $\int_{0}^{b} z^{-p} d z$ where $\mathrm{p}$ is a positive real constant equivalent to $m / 2$. It can be shown that this integral converges if $p<1$ and diverges if $p \geq 1$, which are equivalent to $m \geq 2$. Note that the integral only diverges if the profile extends to $x=0$, so that any truncation will cause the integral to converge. The two solutions for $S(x)$ are provided for the case where $m \neq 2$ (the other two solutions can be obtained using $\left.S_{3,4}(x)=-S_{1,2}(x)\right)$.

$$
\begin{aligned}
S_{1}(x) & =12^{\frac{1}{4}}\left(\epsilon k_{p}\right)^{-\frac{1}{2}}(1-m / 2)^{-1} x^{(1-m / 2)}-\frac{1}{2} 12^{-\frac{1}{4}} k_{y}^{2} \epsilon^{\frac{1}{2}} k_{p}^{-\frac{3}{2}}(1+m / 2)^{-1} x^{(1+m / 2)} \\
& -\frac{1}{8} 12^{-\frac{3}{4}} k_{y}^{4} \epsilon^{\frac{3}{2}} k_{p}^{-\frac{5}{2}}(1+3 m / 2)^{-1} x^{(1+3 m / 2)} \\
S_{2}(x) & =\mathrm{i} 12^{\frac{1}{4}}\left(\epsilon k_{p}\right)^{-\frac{1}{2}}(1-m / 2)^{-1} x^{(1-m / 2)}+\frac{\mathrm{i}}{2} 12^{-\frac{1}{4}} k_{y}^{2} \epsilon^{\frac{1}{2}} k_{p}^{-\frac{3}{2}}(1+m / 2)^{-1} x^{(1+m / 2)} \\
& -\frac{\mathrm{i}}{8} 12^{-\frac{3}{4}} k_{y}^{4} \epsilon^{\frac{3}{2}} k_{p}^{-\frac{5}{2}}(1+3 m / 2)^{-1} x^{(1+3 m / 2)}
\end{aligned}
$$

The terms in Eq. (19) can now be used to calculate the wedge displacement in two dimensions providing at least four boundary conditions are provided, as there are four solutions for $S(x)$ and therefore the Fourier amplitude $B_{j}(x)$ has four solutions. The following section will incorporate these wedge solutions into the plate model.

\subsection{Wedge boundary conditions}

When a wedge is applied to the end of the constant thickness plate, the numerical method now has twelve unknown amplitudes rather than eight. Therefore the matrices are adjusted so that $\mathbf{W}$ and $\mathbf{A}$ are twelve by one. The arrangement of the Fourier amplitudes are now $\mathbf{A}=\left[A_{j}^{-}, A_{j}^{+}, B_{j}\right]^{T}$. 
Joining the wedge to the constant thickness plate modifies the first two boundary conditions, where the terms for the wedge displacement are obtained from the equations in the previous section and Eq. (14)

Boundary condition 1, 2: At $x=a$, specify continuity of displacement and slope.

$$
\begin{gathered}
w^{-}(x, \omega)-w_{w}(x, \omega)=0 \text { at } x=a . \\
\frac{\partial w^{-}(x, \omega)}{\partial x}-\frac{\partial w_{w}(x, \omega)}{\partial x}=0 \text { at } x=a .
\end{gathered}
$$

The additional four boundary conditions can then be written as;

Boundary condition 9: At $x=a$, specify continuity of second and third derivatives.

$$
\begin{aligned}
& \frac{\partial^{2} w^{-}(x, \omega)}{\partial x^{2}}-\frac{\partial^{2} w_{w}(x, \omega)}{\partial x^{2}}=0 \text { at } x=a . \\
& \frac{\partial^{3} w^{-}(x, \omega)}{\partial x^{3}}-\frac{\partial^{3} w_{w}(x, \omega)}{\partial x^{3}}=0 \text { at } x=a .
\end{aligned}
$$

At the truncation point of the wedge, it is assumed that the plate ends with a free edge (although a very thin point), providing two final conditions.

Boundary condition 11, 12: At $x=x_{t}$, specify bending moment and shear force.

$$
\begin{gathered}
\frac{\partial^{2} w_{w}(x, \omega)}{\partial x^{2}}-\nu k_{y}^{2} w_{w}(x, \omega)=0 \text { at } x=x_{t} . \\
\frac{\partial^{3} w_{w}(x, \omega)}{\partial x^{3}}-(2-\nu) k_{y}^{2} \frac{\partial w_{w}(x, \omega)}{\partial x}=0 \text { at } x=x_{t} .
\end{gathered}
$$

The Fourier transforms of the boundary conditions are taken and implemented using the full derivative equations, shown for all matrices in the Appendix for completeness.

\subsection{Damping in a plate of variable thickness}

In order to compare experimental measurements and numerical predictions, it is necessary to include a small amount of damping into the plate, to account for natural damping in the materials. In addition, it is necessary to be able to predict the change in material damping when additional damping layers are applied to the plate or wedge surface.

The loss factor of a material $\eta$ is introduced through a complex Young's modulus in the bending stiffness, $E=E(1+\eta \mathrm{i})$. It is well known that if a layer of viscoelastic material is applied on one surface of a plate with loss factor $\eta_{D}$, it can alter the overall loss factor of such a laminated composite plate, $\eta_{\text {comp }}$ as shown in Ref [3]. If the thickness of the damping layer is $h_{D}$ with loss factor 
$\eta_{D}$ and Young's modulus $E_{D}$, the authors of Ref [3] quote results by Oberst where the composite damping is given as,

$$
\eta_{\text {comp }}=\frac{\eta_{D} \alpha\left[12 \beta^{2}+\Gamma^{2}\left(1+\alpha^{2}\right)\right]}{[1+\alpha]\left[12 \alpha \beta^{2}+(1+\alpha)\left(1+\alpha \Gamma^{2}\right)\right]},
$$

where $\Gamma=h_{D} / h_{w}, \alpha=\Gamma E_{D} / E w$ and $\beta=\left(h_{D}+h_{w}\right) / 2 h_{w}$. If the extensional stiffness of the damping layer is significantly less than the wedge layer, $\alpha<1$, then terms in $\alpha^{2}$ and higher may be neglected, to obtain,

$$
\eta_{\mathrm{comp}}=\frac{\eta_{D} \frac{E_{D} h_{D}}{E_{w} h_{w}}\left[4\left(\frac{h_{D}}{h_{w}}\right)^{2}+6\left(\frac{h_{D}}{h_{w}}\right)+3\right]}{1+\frac{E_{D} h_{D}}{E_{w} h_{w}}\left[4\left(\frac{h_{D}}{h_{w}}\right)^{2}+6\left(\frac{h_{D}}{h_{w}}\right)+5\right]} .
$$

This expression and simplified forms have been used by Krylov et al. [7][8] to analyse damping of flexural waves in laminated wedges with power-law profiles $m=2, m=3$ and $m=4$. Here the same approach is used, in particular for a wedge with a power-law $m=2.2$.

For a wedge of profile $m=2.2$ with a damping layer applied, the composite damping ratio is shown in Fig. 4. Note that the composite damping only rises significantly at the very end of the wedge, when the wedge thickness becomes small. At this point the composite damping rises quickly, for even thin damping layers, showing the critical advantage of damping tapered wedges.

In comparisons with experimental measurements, a strip of damping tape with approximate parameters $\eta_{D}=0.2$, of width $2 \mathrm{~cm}$ and $E_{D}=10 \mathrm{MPa}$ yields a composite damping of $1.62 \%$ for a wedge of profile $m=2.2$ and $0.91 \%$ for one with a profile $m=3$. The former plot is shown in Fig. 5 where a simple average based on the integrated reflection coefficient is used to find the overall damping in the wedge.

A layer of damping tape applied to a thick plate will have relatively little impact, which is why the addition of a truncated power-law wedge with damping is so advantageous. In the following section, the results from the numerical model will be compared to experimental measurements.

\section{Experimental investigations}

This section provides a description of the manufacturing method used to produce three rectangular plates, two of which incorporate wedge profiles. A com- 
parison is then undertaken between the experimental measurements on these plates and predictions generated using the equations in the previous section.

The results presented in this paper utilise only one measurement point for the acceleration on the plate. At any given frequency, the amplitude distribution of the plate can be separated into nodal and anti-node points based on their position. If the damping is assessed at only a few discrete frequencies, there exists the possibility that the location of the accelerometer will line up with an anti-node, creating a misleading impression of the amplitude change. To mitigate against the chances of this occurring, the damping is assessed by examination of the vibration amplitude for a broad frequency range. At lower frequencies the individual modes will dominate the response of the plate, whereas at higher frequencies the modes are so close together that they effectively merge. As it is not expected that the damped wedge will attenuate much energy at lower frequencies (the flexural wavelength is longer than the wedge length), we do not comment strongly on these lower frequencies, instead concentrating on the trend behaviour for the upper frequency range.

The assessment of the damping will be carried out in two ways. In presenting the results, certain upper frequencies are selected where the peak amplitude of the point mobility at a resonance is noted in the text (comparing the amplitude of one plate against another at the same frequency). It is expected that the damping method presented in this paper will reduce the peak amplitude of any resonance. Therefore, a summary table is also provided showing the average amplitude reduction in a given frequency band.

The comparisons for a simple rectangular plate, with and without a single layer of damping material are provided in section 3.2. Then, this simple plate is compared with one incorporating a wedge in section 3.3. Finally, in section 3.4 , the response of a plate with a wedge when damped and undamped is examined.

\subsection{Manufacturing method}

A key requirement of any application of the plate incorporating a damped tapered wedge is that the manufacturing method should be relatively simple and cost effective. Previous research has shown that it is possible to obtain a 1-D wedge at one end of a plate using wire erosion [9], although this is time consuming and costly. The plates used in the experiments described in this paper are milled from plate steel by traversing the cutting bit along the length of the plate fifty times at the wedge, each of a slightly deeper cut and closer to the edge of the plate. As can be seen in the illustration, Fig. 6, when close to the end, the material is extremely thin such that the cutter tends to grab, 
causing a non-uniform truncation point. In addition, the heat caused by the rotation of the tool-bit on the metal causes the thin surface to curl up at the truncation point. Although the wedge profile is comprised of discrete steps, it is assumed that this may be directly comparable to the case of a smooth tapered surface.

Three plates have been produced from a single sheet of cold rolled steel (thickness $5.04 \mathrm{~mm}$ ) to ensure all material properties are uniform. A simple plate of dimension 280x195 mm (and no wedge) has been tested and compared with a similar plate with a wedge profile following an $\mathrm{m}=2.2$ shape of overall length $50 \mathrm{~mm}$ (the distance from the truncation point to the constant thickness plate being $45 \mathrm{~mm}$ ). A separate plate with a wedge profile following an $\mathrm{m}=3.0$ shape of overall length $50 \mathrm{~mm}$ (the distance from the truncation point to the constant thickness plate is $37 \mathrm{~mm}$ ) has also been manufactured.

As detailed in section 2.3, the theoretical acoustic black hole effect only occurs for profiles which are designed with a value of $m \geq 2$. In order to ensure that the test specimens are inside this range, the first wedge profile is designed with a value of $m=2.2$, to account for any machining tolerances. This also takes into account any additional differences caused by the profile surface being stepped rather than smooth. However, in theory there should be little difference between a truncated plate with profile $m=2.0$ and $m=2.2$.

Each plate has been tested using the same supporting frame, consisting of small straps placed along the two of the plate edges, see Fig. 7 and subsequently discussed. An electromagnetic shaker fed by a broadband signal is attached to the plate underside through a force transducer (B\&K 8200) with an accelerometer on the top surface (B\&K Type 4371), both held using wax. Two charge amplifiers $(B \& K)$ provide amplification for the signal prior to the individual time histories being logged (the maximum sampling frequency being $24 \mathrm{kHz}$ ). A validated fast Fourier transform routine was used to obtain the average driving and cross point mobilities relating the normal velocity of the plate surface to the applied force (approximately fifty averages were used in the post processing of the time signals). To obtain the velocity $\dot{w}(\omega)$ from the acceleration $\ddot{w}(\omega)$, the relation $\dot{w}(\omega)=-\mathrm{i} \ddot{w}(\omega) / \omega$ is applied.

The locations of the force transducer and accelerometer on the plate surface are the same as those described for the numerical method in section 2.2. The sampling frequency chosen for the experimental measurements also matched the numerical method, at $10 \mathrm{~Hz}$ intervals. Only a thin layer of wax attached the accelerometer and force transducers, with a low amplitude of vibration used to ensure the measurements were valid.

The experimental results show that the signal to noise ratio is highly variable. Above a frequency of approximately $5 \mathrm{kHz}$, there are reductions in the 
quality of the experimental results and above $7 \mathrm{kHz}$ the errors increase significantly. Much of this variability is due to the implementations of the boundary conditions, noise within the equipment and attachment method.

In order to study the addition of damping to the plates, a single strip of damping tape was applied along the length of the wedge, closest to the truncation position. It is assumed that the tape and the adherence is uniform.

\subsection{Comparison of experimental and numerical methods}

The driving point mobilities for a simple plate without any wedge profile are shown in Fig. 8 for numerical predictions and experimental measurements as functions of frequency. The amplitude of the mobility response is plotted in $\mathrm{dB}$ with a reference value of $1 \mathrm{~ms}^{-1} \mathrm{~N}^{-1}$.

Many of the mobility response peaks are located at frequencies relatively close to those predicted by the numerical plate model. The first resonance is accurately found at $350 \mathrm{~Hz}$ and for many of the resonant frequencies below $4 \mathrm{kHz}$, the differences are no more than $100 \mathrm{~Hz}$, except the resonance at $2 \mathrm{kHz}$ which is not well represented. The overall reduction in amplitude with frequency is matched by the numerical model, leading to the conclusion that it is possible to use this as a base for further comparisons.

There are, however, significant differences in the comparison for frequencies between $3.7-7 \mathrm{kHz}$, where the experimental measurements are not regularly distributed. At the top of this range, there are no signs of resonances or antiresonances, therefore it is difficult to establish whether these are modes of vibration in the $x$ or $y$ directions which are suffering from damping due to the imperfect implementation of the boundary conditions. The numerical model assumes that the side edges of the plate are simply supported, along the entire length, whereas the experimental method only constrains the plate displacement at the edge at a select few discrete points. Therefore, although there are frequencies where resonances coincide, it is possible that the mode shapes are not necessarily similar and therefore the specific attenuation at that frequency would not necessarily be the same. However, the numerical results indicate the typical levels of attenuation that may appear for a given mode and provide indicative damping levels for a broad frequency range. Therefore, although the boundary conditions are not necessarily compatible, the damping trends are independent of these side constraints, such that the application of damped, tapered wedges can be evaluated for different side conditions. Further direct comparisons between the numerical and experimental measurements will be minimised, in favour of observing the trends that each show.

In order to quantify the effect of damping material when applied to the plates 
with a wedge profile, the same damping tape is applied to the simple rectangular plate and compared with the undamped response. It is expected that when the tape is applied that any changes will be minimal, as the thickness of the tape (approximately $1 \mathrm{~mm}$ ) is much thinner than the plate and the stiffness is significantly reduced. The numerical driving point mobility predictions for a simple plate with and without a layer of damping tape are shown in Fig. 9(a), with the corresponding experimental measurements in Fig. 9(b). In both cases the damped plates are represented by the dashed lines. As expected, the location of the vibration resonances is not altered as the tape has negligible stiffness, however, there is a reduction in peak amplitudes of approximately 5-6 $\mathrm{dB}$ (for example, at the resonance at $3 \mathrm{kHz}$, the numerical prediction of the peak mobility amplitude for the undamped plate is $-42 \mathrm{~dB}$, while the damped plate is $-46 \mathrm{~dB})$. The actual reduction found in the experimental results is variable, ranging from 1 to $5 \mathrm{~dB}$, depending on the frequency. At a frequency of $3 \mathrm{kHz}$, the undamped amplitude is $-36 \mathrm{~dB}$ while the damped amplitude is $-39 \mathrm{~dB}$, whereas at $5.75 \mathrm{kHz}$ the respective amplitudes are $-41.5 \mathrm{~dB}$ and $-42.5 \mathrm{~dB}$.

The cross point mobility results for the numerical and experimental measurements are shown in Figs. 10(a) and (b) respectively. The overall amplitudes are approximately $10 \mathrm{~dB}$ less than the driving point mobility resonances. Again, there are several modes of vibration which are well represented by the numerical model, however there are also frequencies $(3-4.5 \mathrm{kHz})$ which do not agree well. As the accelerometer is placed in the offset position, it measures more of a mixture of odd and even modes of vibration and may be more affected by the imperfect boundary conditions on the edge of the plate. The addition of a single layer of damping tape does not significantly affect the amplitude response.

\subsection{Comparison of a simple plate with the addition of a wedge profile}

The previous section has provided a comparison of simple rectangular plates when undamped and when a single layer of damping tape is applied. In this section, the comparison will be made between a simple plate and one which has a profiled wedge.

In section 2.3, it has been shown that according to simplified bending plate theory, a wave which travels into a wedge profile (with $m \geq 2$ ) finds that it's propagation speed slows down (in addition the amplitude increases). When a truncation of the wedge is introduced, the wave is entirely reflected back from the edge and then increases in propagation speed and decreases in amplitude. Since the experimental plates do indeed have a truncation on the wedge shape, it is expected that the location of the resonances will move significantly due 
to the increased plate length, but the overall amplitude will not, as there is no more inherent damping than in the simple plate.

The driving point mobility response of a plate which has a profile of $m=2.2$ is compared to the simple rectangular plate in Fig. 11 for (a) numerical predictions and (b) experimental measurements. The location of the frequencies of resonance are shifted significantly, especially above $2 \mathrm{kHz}$. The amplitude of the waves in the experimental results show some reduction above a frequency of $6 \mathrm{kHz}$, although the signal to noise ratio of the measurements has noticeably decreased.

At a frequency of $2.9-3 \mathrm{kHz}$, the numerical prediction of the peak mobility amplitude of the plain plate is $-42 \mathrm{~dB}$, whereas the plate with wedge attachment is $-46 \mathrm{~dB}$ (a difference of $4.0 \mathrm{~dB}$ ). At a higher frequency, e.g. $5.5 \mathrm{kHz}$, the difference is still only $0.5 \mathrm{~dB}$, so it may be concluded that attaching an undamped wedge does not provide a significant reduction in peak mobility amplitudes.

With a profile such as $m=3.0$, the thickness decreases at a faster rate as $x \rightarrow$ 0 . The theory for a wedge with no truncation would indicate that the acoustic black hole effect would be more pronounced in this case. This comparison is shown in Fig. 12 where the plate with a wedge profile of $m=3.0$ is directly compared to a simple rectangular plate (once again the numerical results are shown in subplot (a) and experimental results in (b)).

It may be seen that although the locations of the frequency resonances are shifted again, the amplitude does not decrease as for higher excitation frequencies. This may be partly explained by the location of the truncation point. As the material becomes so thin so soon after starting the wedge profile, the relative truncation point occurs sooner than for the $m=2.2$ profile. Further analysis is provided in the summary tables at the end of this section.

\subsection{Response of plates with wedge profiles when damped}

As there are difficulties manufacturing a wedge shape without any truncation, attention is now placed on absorbing the plate vibration energy in a more efficient manner. While the waves are inside the wedge, the amplitude grows and their propagation speed is reduced. A damping layer applied to the wedge, which if not stiff or thick in comparison to the wedge material, should be able to transfer the flexural kinetic energy of the surface to heat better than for the case where the damping layer is applied to the simple plate.

The comparison of a plate with a profile of power-law $\mathrm{m}=2.2$ with and without damping tape applied is shown in Figs. 13(a) and (b) for the numerical 
predictions and experimental measurements respectively.

It can be seen that the amplitude of the resonances at the lowest frequencies are not reduced by the addition of damping tape, as the excitation wavelength in the plate is far longer than the width of the strip of tape. However, for higher frequencies, for example those over $4 \mathrm{kHz}$, there appears to be a significant reduction in the amplitude of the order $7-15 \mathrm{~dB}$. At very high frequencies, the experimental results do not show a significant reduction, however the signal to noise ratio of these measurements has decreased significantly for frequencies above $7 \mathrm{kHz}$.

For example, at a resonance of $2.9-3 \mathrm{kHz}$, the numerical predictions for the peak mobility amplitude for a plate with an undamped wedge are $-46.0 \mathrm{~dB}$. As soon as the damping is applied to the wedge, this decreases to $-55.0 \mathrm{~dB}$ (a difference of $9.0 \mathrm{~dB}$ ). At a higher frequency, $6.5 \mathrm{kHz}$, the respective figures are -52.0 and $-61.0 \mathrm{~dB}$ (a difference of $9.0 \mathrm{~dB}$ ).

The results for a plate with a wedge profile of power-law $m=3.0$ are shown with and without a layer of damping tape in Fig. 14(a) and (b) for the numerical and experimental measurements respectively. Identical trends are shown, with the plate with damping layer showing a lower peak mobility amplitude between peaks and troughs above $2 \mathrm{kHz}$, a reduction of between 5-12 dB. The measurements above $6.5 \mathrm{kHz}$ are not of sufficient quality to resolve the changes.

Examples of the peak mobility measurements from the numerical predictions are as follows; at a frequency of 2.9-3.0 kHz, the plate with an undamped wedge of profile $m=3$ has a peak amplitude of $-48 \mathrm{~dB}$, whereas the plate with the damped wedge is $-56.25 \mathrm{~dB}$ (a reduction of $8.25 \mathrm{~dB}$ ). At a higher frequency of $6.2 \mathrm{kHz}$, the respective figures are -51.0 and $-64.0 \mathrm{~dB}$ (a difference of $13 \mathrm{~dB}$ ).

A summary table is provided, showing the average peak mobility found in three different frequency bands. This mean average mobility is calculated by identifying the peak amplitudes of all resonances in the frequency band and then dividing by the number of resonances. Although this does not take into account the energy in each band, it does provide a useful measure of the effectiveness of the different damping treatments, as most practical implementations are interested in reductions of peak mobility.

The summary of the average peak mobility predictions for the numerical predictions are shown in Table 1. It can be seen that the addition of an undamped wedge is almost as useful as applying the damping directly onto the constant thickness plate. However, when the damping is applied to the wedge, the amplitude reductions are more significant. Interestingly, the numerical predictions do indicate that the $m=3$ profile should still be more effective. 
The corresponding average peak mobility summary for the experimental measurements can be seen in Table 2. In this case the reductions for a wedge of profile $m=3$ are not as effective as for the $m=2.2$ case, whether damped or undamped.

It has been shown that a wedge attachment of profile $m \geq 2$ can significantly change the frequency location of resonances. The profile $m=2.2$ appears to offer a greater advantage in terms of minimising the undamped vibration than the $m=3.0$ wedge, as the manufacturing technique truncates the wedge too soon for the latter case. The case of $m=2.2$ profile appears to have more space to allow the black hole effect to occur.

Utilising a combination of a wedge profile in which the thickness decreases and a damping material allows a greater proportion of flexural vibration energy to be attenuated than if the damping material is applied directly to a thick plate.

\section{Conclusions}

A numerical model for a finite plate with wedge attachments has been developed which can predict natural frequencies of vibration for finite width plates up to a range of $7 \mathrm{kHz}$. This has been validated against experimental measurements. Although there are some differences, these are partly due to the applied boundary conditions on the plate.

The numerical model has been used to compare three plates: a rectangular plate with no wedge attachment, one plate with a $m=2.2$ power-law profile wedge and one with a $m=3.0$ profile wedge. The numerical model was able to successfully predict the location and amplitudes of the natural frequencies of vibration. Similar experimental measurements have been made on three plates for comparison.

It has been shown for the above-mentioned plate structures with wedge attachments that a combination of a wedge shape of power-law profile and small amounts of damping materials can lead to large decreases in the amplitude of resonances in thick plates.

The addition of an undamped wedge to a plate significantly changes the location of the frequency resonances but does not significantly change the amplitude of the resonances. It is equivalent to extending the plate so that a mixture of odd and even modes are found. Note that the change in thickness at the edges also increases the amplitude of vibration on the wedge surface. 
Damping material applied to all plates shows that a 1-5 dB reduction in peak amplitudes is possible when the damping material is applied to the plain plate. This is a small change as the thickness and stiffness of the tape is negligible when compared to the thickness of the steel.

When the damping material is applied to the plates with the wedge addition, reductions of approximately $10-15 \mathrm{~dB}$ are possible for mid-range frequencies, as the tape stiffness and thickness are comparable to the wedge thickness close to the truncation points. It is found that the wedge with a power-law profile $m=2.2$ performs more efficiently than $m=3.0$, as the truncation point occurs later.

It is important to match the parameters of the damping material to the thickness and extensional stiffness of the plate to maximise attenuation. Applying thick damping material to a wedge will not necessarily result in further damping. The thickness and stiffness can be optimised on the wedge to maximise damping performance.

\section{Acknowledgements}

The research reported here has been supported by EPSRC grant EP/F009232/1. 


\section{Appendix: Terms for a rectangular plate with a wedge addition}

The matrix terms for a rectangular plate with a wedge addition are provided here for completeness. The exponential terms in $y$ and $t$ have been omitted from these terms to minimise duplication.

The matrix $\mathbf{A}$ is twelve rows by one column $(12,1)$. The terms are,

$$
\begin{aligned}
& A[1]=A_{1}^{-}, \quad A[2]=A_{2}^{-}, \quad A[3]=A_{3}^{-}, \quad A[4]=A_{4}^{-} \\
& A[5]=A_{1}^{+}, A[6]=A_{2}^{+}, \quad A[7]=A_{3}^{+}, \quad A[8]=A_{4}^{+} \\
& A[9]=B_{1}, A[10]=B_{2}, A[11]=B_{3}, A[12]=B_{4}
\end{aligned}
$$

The matrix $\mathbf{W}$ contains the results of the boundary conditions, whose terms are,

$$
\begin{aligned}
& W[1]=0, \quad W[2]=0, \quad W[3]=0, \quad W[4]=0, \\
& W[5]=0, \quad W[6]=0, \quad W[7]=0, \quad W[8]=1, \\
& W[9]=0, W[10]=0, W[11]=0, W[12]=0
\end{aligned}
$$

The matrix $\mathbf{X}$ contains the applied boundary conditions, a twelve by twelve in size. The terms are,

$$
\begin{array}{cc}
X[1,1]=k_{1}^{2} e^{k_{1}\left[a-x_{0}\right]}, & X[1,2]=k_{2}^{2} e^{k_{2}\left[a-x_{0}\right]} \\
X[1,3]=k_{3}^{2} e^{k_{3}\left[a-x_{0}\right]}, & X[1,4]=k_{4}^{2} e^{k_{4}\left[a-x_{0}\right]} \\
X[1,9]=-k_{p}^{2} \frac{\partial S_{1}(a)}{\partial x}{ }^{2} e^{\mathrm{i} k_{p} S_{1}(a)}, & X[1,10]=-k_{p}^{2} \frac{\partial S_{2}(a)}{\partial x} e^{\mathrm{i} k_{p} S_{2}(a)} \\
X[1,11]=-k_{p}^{2} \frac{\partial S_{3}(a)}{\partial x} e^{\mathrm{i} k_{p} S_{3}(a)}, & X[1,12]=-k_{p}^{2} \frac{\partial S_{4}(a)}{\partial x} e^{\mathrm{i} k_{p} S_{4}(a)} \\
X[2,1]=k_{1}^{3} e^{k_{1}\left[a-x_{0}\right]}, & X[2,2]=k_{2}^{3} e^{k_{2}\left[a-x_{0}\right]} \\
X[2,3]=k_{3}^{3} e^{k_{3}\left[a-x_{0}\right]}, & X[2,4]=k_{4}^{3} e^{k_{4}\left[a-x_{0}\right]} \\
X[2,9]=-\mathrm{i} k_{p}^{3} \frac{\partial S_{1}(a)}{\partial x} e^{\mathrm{i} k_{p} S_{1}(a)}, & X[2,10]=-\mathrm{i} k_{p}^{3} \frac{\partial S_{2}(a)}{\partial x} e^{\mathrm{i} k_{p} S_{2}(a)} \\
X[2,11]=-\mathrm{i} k_{p}^{3} \frac{\partial S_{3}(a)}{\partial x} e^{\mathrm{i} k_{p} S_{3}(a)}, & X[2,12]=-\mathrm{i} k_{p}^{3} \frac{\partial S_{4}(a)}{\partial x} e^{\mathrm{i} k_{p} S_{4}(a)} \\
X[3,5]=\left(k_{1}^{2}-\nu k_{y}^{2}\right) e^{k_{1}\left[b-x_{0}\right]}, & X[3,6]=\left(k_{2}^{2}-\nu k_{y}^{2}\right) e^{k_{2}\left[b-x_{0}\right]} \\
X[3,7]=\left(k_{3}^{2}-\nu k_{y}^{2}\right) e^{k_{3}\left[b-x_{0}\right]}, & X[3,8]=\left(k_{4}^{2}-\nu k_{y}^{2}\right) e^{k_{4}\left[b-x_{0}\right]} \\
X[4,5]=\left(k_{1}^{3}-(2-\nu) k_{y}^{2} k_{1}\right) e^{k_{1}\left[b-x_{0}\right]}, & X[4,6]=\left(k_{2}^{3}-(2-\nu) k_{y}^{2} k_{2}\right) e^{k_{2}\left[b-x_{0}\right]} \\
X[4,7]=\left(k_{3}^{3}-(2-\nu) k_{y}^{2} k_{3}\right) e^{k_{3}\left[b-x_{0}\right]}, & X[4,8]=\left(k_{4}^{3}-(2-\nu) k_{y}^{2} k_{4}\right) e^{k_{4}\left[b-x_{0}\right]}
\end{array}
$$




$$
\begin{aligned}
& X[5,1]=1, \quad X[5,2]=1, \quad X[5,3]=1, \quad X[5,4]=1 \\
& X[5,5]=-1, \quad X[5,6]=-1, \quad X[5,7]=-1, \quad X[5,8]=-1 \\
& X[6,1]=k_{1}, \quad X[6,2]=k_{2}, \quad X[6,3]=k_{3}, \quad X[6,4]=k_{4} \\
& X[6,5]=-k_{1}, X[6,6]=-k_{2}, X[6,7]=-k_{3}, X[6,8]=-k_{4} \\
& X[7,1]=k_{1}^{2}, \quad X[7,2]=k_{2}^{2}, \quad X[7,3]=k_{3}^{2}, \quad X[7,4]=k_{4}^{2} \\
& X[7,5]=-k_{1}^{2}, X[7,6]=-k_{2}^{2}, X[7,7]=-k_{3}^{2}, X[7,8]=-k_{4}^{2} \\
& X[8,1]=k_{1}^{3}, \quad X[8,2]=k_{2}^{3}, \quad X[8,3]=k_{3}^{3}, \quad X[8,4]=k_{4}^{3} \\
& X[8,5]=-k_{1}^{3}, X[8,6]=-k_{2}^{3}, X[8,7]=-k_{3}^{3}, X[8,8]=-k_{4}^{3} \\
& X[9,1]=e^{k_{1}\left[a-x_{0}\right]}, \quad X[9,2]=e^{k_{2}\left[a-x_{0}\right]} \\
& X[9,3]=e^{k_{3}\left[a-x_{0}\right]}, \quad X[9,4]=e^{k_{4}\left[a-x_{0}\right]} \\
& X[9,9]=-e^{\mathrm{i} k_{p} S_{1}(a)}, \quad X[9,10]=-e^{\mathrm{i} k_{p} S_{2}(a)} \\
& X[9,11]=-e^{\mathrm{i} k_{p} S_{3}(a)}, X[9,12]=-e^{\mathrm{i} k_{p} S_{4}(a)} \\
& X[10,1]=k_{1} e^{k_{1}\left[a-x_{0}\right]}, \quad X[10,2]=k_{2} e^{k_{2}\left[a-x_{0}\right]} \\
& X[10,3]=k_{3} e^{k_{3}\left[a-x_{0}\right]}, \quad X[10,4]=k_{4} e^{k_{4}\left[a-x_{0}\right]} \\
& X[10,9]=-\mathrm{i} k_{p} \frac{\partial S_{1}(a)}{\partial x} e^{\mathrm{i} k_{p} S_{1}(a)}, \quad X[10,10]=-\mathrm{i} k_{p} \frac{\partial S_{2}(a)}{\partial x} e^{\mathrm{i} k_{p} S_{2}(a)} \\
& X[10,11]=-\mathrm{i} k_{p} \frac{\partial S_{3}(a)}{\partial x} e^{\mathrm{i} k_{p} S_{3}(a)}, X[10,12]=-\mathrm{i} k_{p} \frac{\partial S_{4}(a)}{\partial x} e^{\mathrm{i} k_{p} S_{4}(a)} \\
& X[11,9]=\left(-k_{p}^{2}{\frac{\partial S_{1}\left(x_{t}\right)^{2}}{\partial x}}^{2}-\nu k_{y}^{2}\right) e^{\mathrm{i} k_{p} S_{1}\left(x_{t}\right)}, X[11,10]=\left(-k_{p}^{2}{\frac{\partial S_{2}\left(x_{t}\right)^{2}}{\partial x}}^{2}-\nu k_{y}^{2}\right) e^{\mathrm{i} k_{p} S_{2}\left(x_{t}\right)} \\
& X[11,11]=\left(-k_{p}^{2}{\frac{\partial S_{3}\left(x_{t}\right)}{\partial x}}^{2}-\nu k_{y}^{2}\right) e^{\mathrm{i} k_{p} S_{3}\left(x_{t}\right)}, X[11,12]=\left(-k_{p}^{2} \frac{\partial S_{4}\left(x_{t}\right)^{2}}{\partial x}-\nu k_{y}^{2}\right) e^{\mathrm{i} k_{p} S_{4}\left(x_{t}\right)} \\
& X[12,9]=\left(-\mathrm{i} k_{p}^{3}{\frac{\partial S_{1}\left(x_{t}\right)^{3}}{\partial x}}^{3}-(2-\nu) k_{y}^{2} \mathrm{i} k_{p} \frac{\partial S_{1}\left(x_{t}\right)}{\partial x}\right) e^{\mathrm{i} k_{p} S_{1}\left(x_{t}\right)}, \\
& X[12,10]=\left(-\mathrm{i} k_{p}^{3}{\frac{\partial S_{2}\left(x_{t}\right)}{\partial x}}^{3}-(2-\nu) k_{y}^{2} \mathrm{i} k_{p} \frac{\partial S_{2}\left(x_{t}\right)}{\partial x}\right) e^{\mathrm{i} k_{p} S_{2}\left(x_{t}\right)}, \\
& X[12,11]=\left(-\mathrm{i} k_{p}^{3}{\frac{\partial S_{3}\left(x_{t}\right)}{\partial x}}^{3}-(2-\nu) k_{y}^{2} \mathrm{i} k_{p} \frac{\partial S_{3}\left(x_{t}\right)}{\partial x}\right) e^{\mathrm{i} k_{p} S_{3}\left(x_{t}\right)}, \\
& X[12,12]=\left(-\mathrm{i} k_{p}^{3} \frac{\partial S_{4}\left(x_{t}\right)^{3}}{\partial x}-(2-\nu) k_{y}^{2} \mathrm{i} k_{p} \frac{\partial S_{4}\left(x_{t}\right)}{\partial x}\right) e^{\mathrm{i} k_{p} S_{4}\left(x_{t}\right)}
\end{aligned}
$$

All other terms in $\mathbf{X}$ are null.

\section{References}

[1] M. Heckl, L. Cremer, E. E. Ungar, Structure Borne Sound, 2nd Edition, Springer-Verlag, 1988.

[2] D. J. Mead, Passive Vibration Control, Wiley \& Sons, Chichester, 1998. 
[3] D. Ross, E. M. Kerwin, E. E. Ungar, Damping of plate flexural vibrations by means of viscoelastic laminae, in Structural Damping (Ruzicka, J. E., ed) 3 (1959) 44-87.

[4] C. Vemula, A. N. Norris, G. D. Cody, Attenuation of waves in plates and bars using a graded impedance interface at edges, Journal of Sound and Vibration 196 (1) (1996) 107-127.

[5] M. A. Mironov, Propagation of a flexural wave in a plate whose thickness decreases smoothly to zero in a finite interval, Soviet Physics - Acoustics 34 (1988) 318-319.

[6] V. V. Krylov, Laminated plates of variable thickness as effective absorbers for flexural vibrations, in: Proceedings of the 17th ICA, Rome, Vol. 1, 2001, pp. 270-271.

[7] V. V. Krylov, F. J. B. S. Tilman, Acoustic 'black holes' for flexural waves as effective vibration dampers, Journal of Sound and Vibration 274 (3-5) (2004) 605-619.

[8] V. V. Krylov, New type of vibration dampers utilising the effect of acoustic 'black holes', Acta Acustica united with Acustica 90 (5) (2004) 830-837.

[9] V. V. Krylov, R. E. T. B. Winward, Experimental investigation of the acoustic black hole effect for flexural waves in tapered plates, Journal of Sound and Vibration 300 (1) (2007) 43-49.

[10] K. F. Graff, Wave Motion in Elastic Solids, Clarendon Press, Oxford, 1975.

[11] V. V. Krylov, Geometrical-acoustics approach to the description of localized vibrational modes of an elastic solid wedge, Soviet Physics - Technical Physics 35 (1990) 137-140. 


\section{List of figure captions}

Fig. 1. Illustration of a plate incorporating a wedge of power-law profile.

Fig. 2. Plate model for mobility predictions. Two short plates are joined together with a point excitation force applied as a shear force at the join. At one end is located a wedge with a power-law profile, terminating at the truncation point.

Fig. 3. Wedge profile.

Fig. 4. Variation in composite damping ratio with damping layer thickness and wedge position (wedge profile is $m=2.2$ ).

Fig. 5. Composite damping for a strip of damping material applied to a profile wedge, for comparison to experimental measurements. Wedge profile is $m=2.2$ ).

Fig. 6. Photo showing one of the plates with an $\mathrm{m}=2.2$ profile wedge. The actual wedge shape is displayed, composed of a series of small steps created as part of the milling process. Also note the small variations in truncation position caused by the ripping of the thin metal.

Fig. 7. Experimental apparatus; the plate rests on straps, with the electromagnetic shaker attached to the underside with wax.

Fig. 8. Driving point mobilities for a simple plate, $(|\dot{w} / p|) \mathrm{dB}$ re $1 \mathrm{~m} / \mathrm{s} / \mathrm{N}$; Numerical predictions and experimental measurements.

Fig. 9. Comparison of driving point mobilities for a simple plate, $(|\dot{w} / p|) \mathrm{dB}$ re $1 \mathrm{~m} / \mathrm{s} / \mathrm{N}$; (a) numerical calculations and (b) experimental measurements. Also shown are the mobilities when a single layer of damping tape is applied to the end of the plate. 
Fig. 10. Comparison of the cross point mobilities for a simple plate, $(|\dot{w} / p|) \mathrm{dB}$ re $1 \mathrm{~m} / \mathrm{s} / \mathrm{N}$; (a) numerical calculations and (b) experimental measurements. Also shown are the mobilities when a single layer of damping tape is applied to the end of the plate.

Fig. 11. Comparison of driving point mobilities, $(|\dot{w} / p|) \mathrm{dB}$ re $1 \mathrm{~m} / \mathrm{s} / \mathrm{N}$; (a) numerical calculations and (b) experimental measurements for a simple plate against a plate with a wedge shape using a $\mathrm{m}=2.2$ power-law profile.

Fig. 12. Comparison of driving point mobilities for a simple plate against one with a profile of $\mathrm{m}=3.0$ power-law, $(|\dot{w} / p|) \mathrm{dB}$ re $1 \mathrm{~m} / \mathrm{s} / \mathrm{N}$; (a) numerical predictions and (b) experimental measurements.

Fig. 13. Driving point mobilities for a plate with a wedge profile of power-law $\mathrm{m}=2.2$ without and with a single layer of damping tape applied; (a) numerical predictions and (b) experimental measurements.

Fig. 14. Driving point mobilities for a plate with a wedge profile of power-law $\mathrm{m}=3.0$ without and with a single layer of damping tape applied; (a) numerical predictions and (b) experimental measurements. 


\section{List of figures and captions}

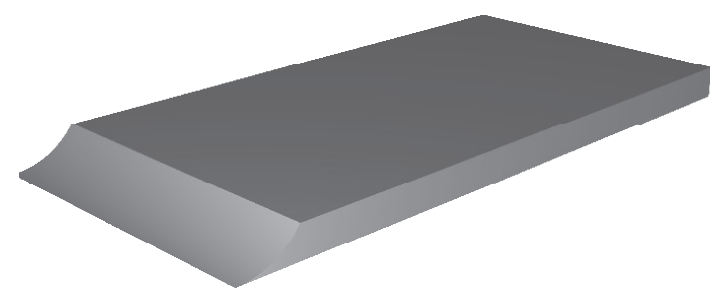

Fig. 1. Illustration of a plate incorporating a wedge of power-law profile. 


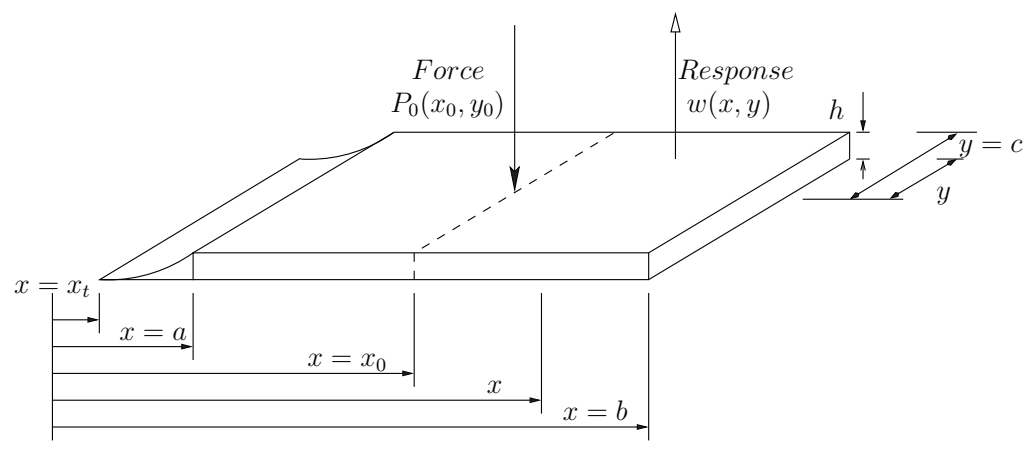

Fig. 2. Plate model for mobility predictions. Two short plates are joined together with a point excitation force applied as a shear force at the join. At one end is located a wedge with a power-law profile, terminating at the truncation point. 


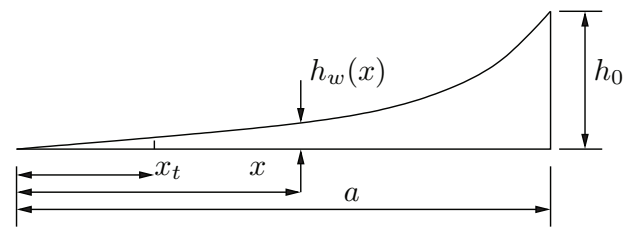

Fig. 3. Wedge profile. 


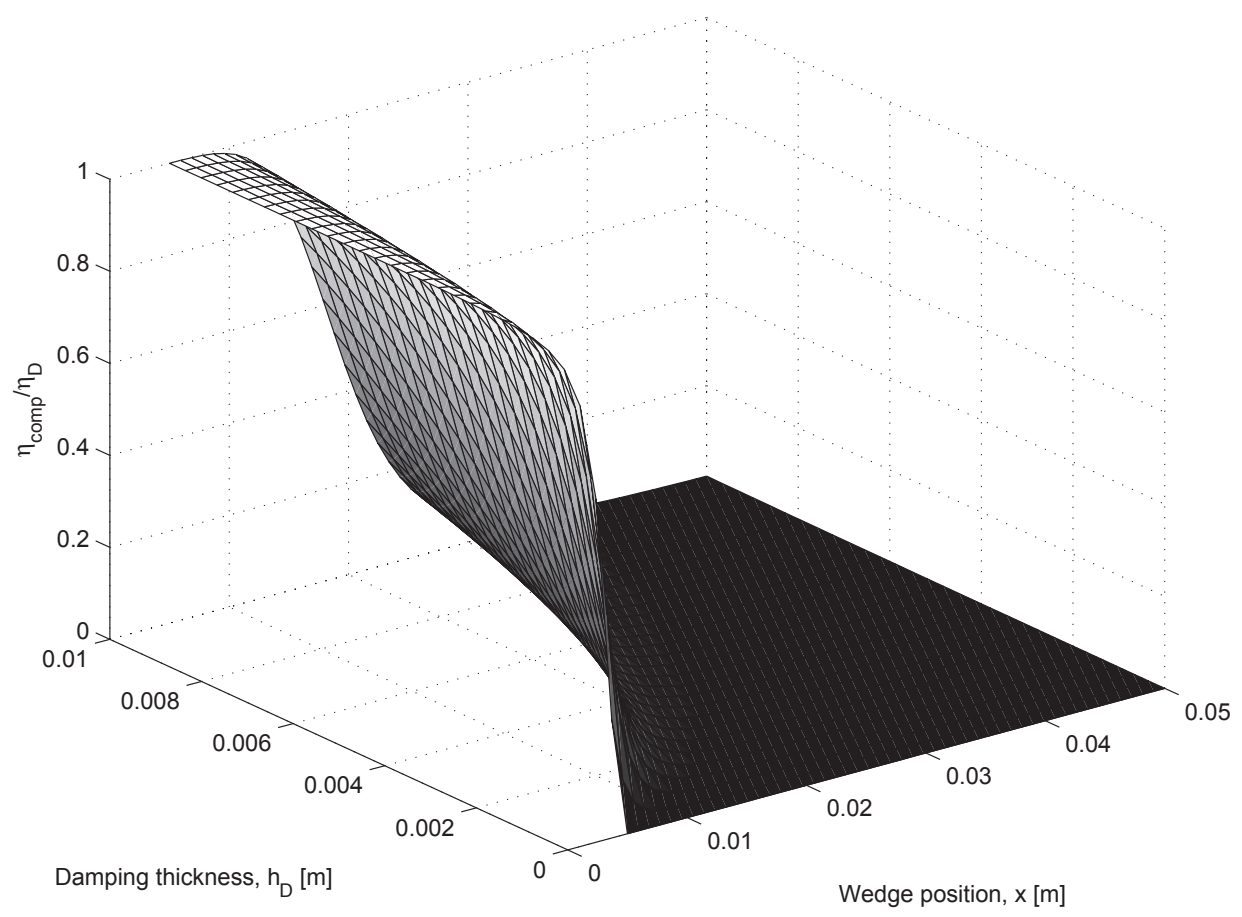

Fig. 4. Variation in composite damping ratio with damping layer thickness and wedge position (wedge profile is $m=2.2$ ). 


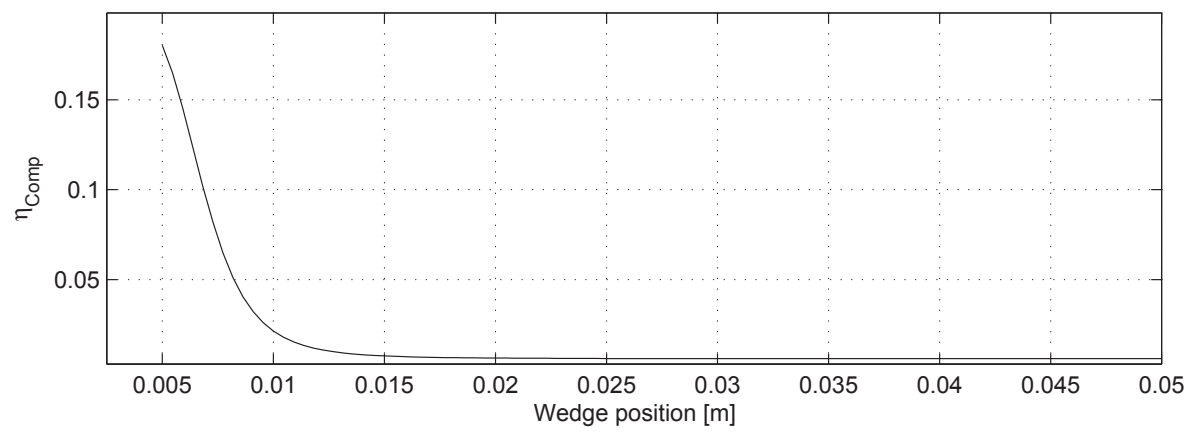

Fig. 5. Composite damping for a strip of damping material applied to a profile wedge, for comparison to experimental measurements. Wedge profile is $m=2.2$ ). 


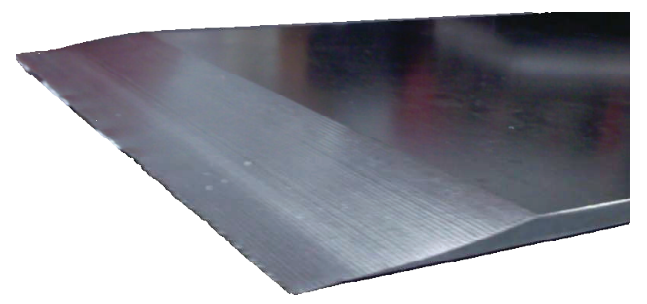

Fig. 6. Photo showing one of the plates with an $\mathrm{m}=2.2$ profile wedge. The actual wedge shape is displayed, composed of a series of small steps created as part of the milling process. Also note the small variations in truncation position caused by the ripping of the thin metal. 


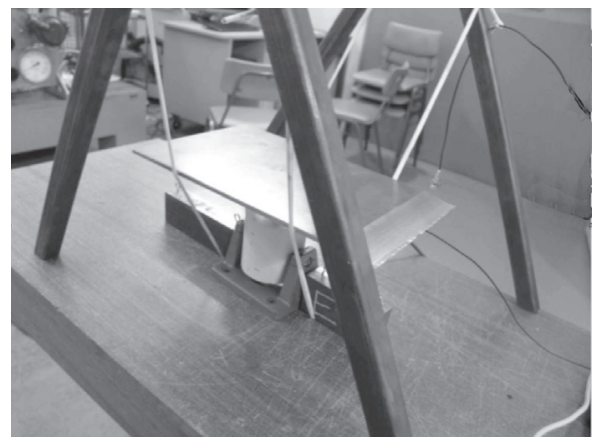

Fig. 7. Experimental apparatus; the plate rests on straps, with the electromagnetic shaker attached to the underside with wax. 


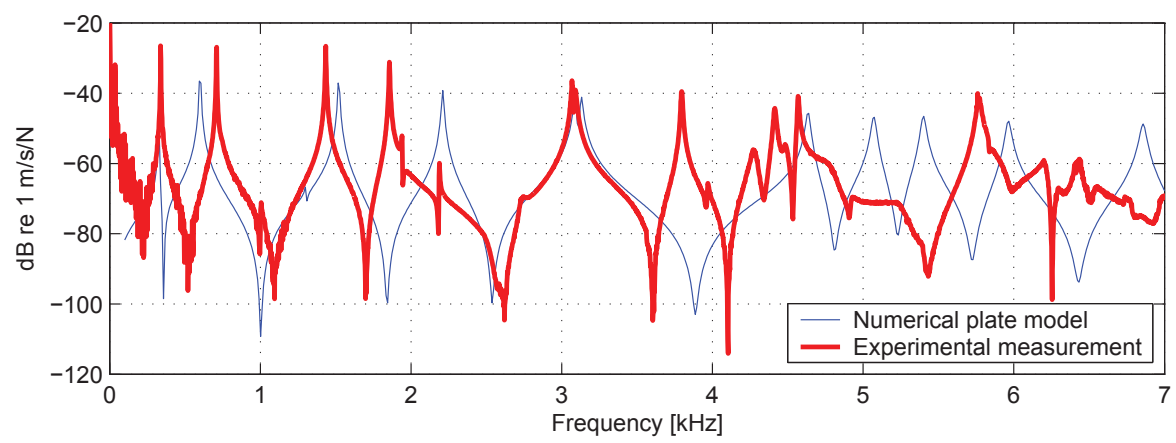

Fig. 8. Driving point mobilities for a simple plate, $(|\dot{w} / p|) \mathrm{dB}$ re $1 \mathrm{~m} / \mathrm{s} / \mathrm{N}$; Numerical predictions and experimental measurements. 


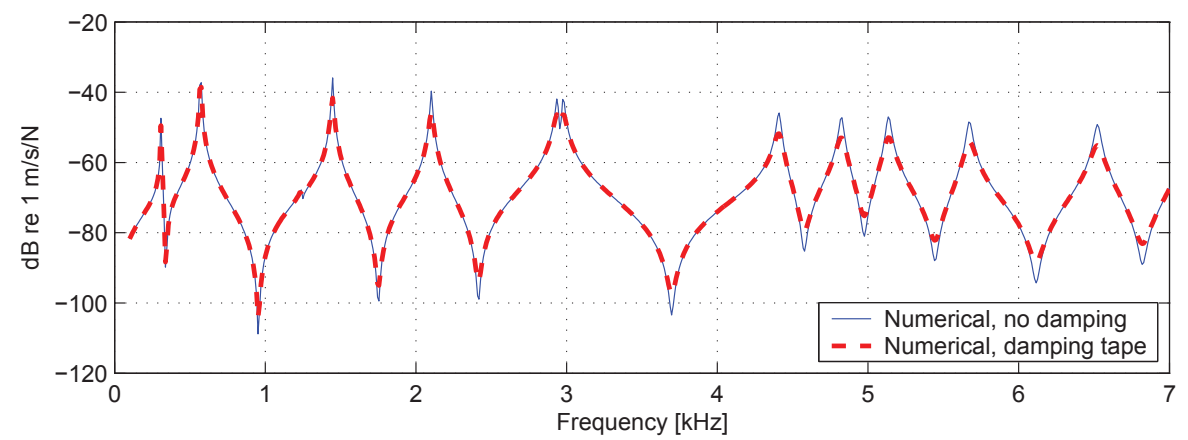

(a)

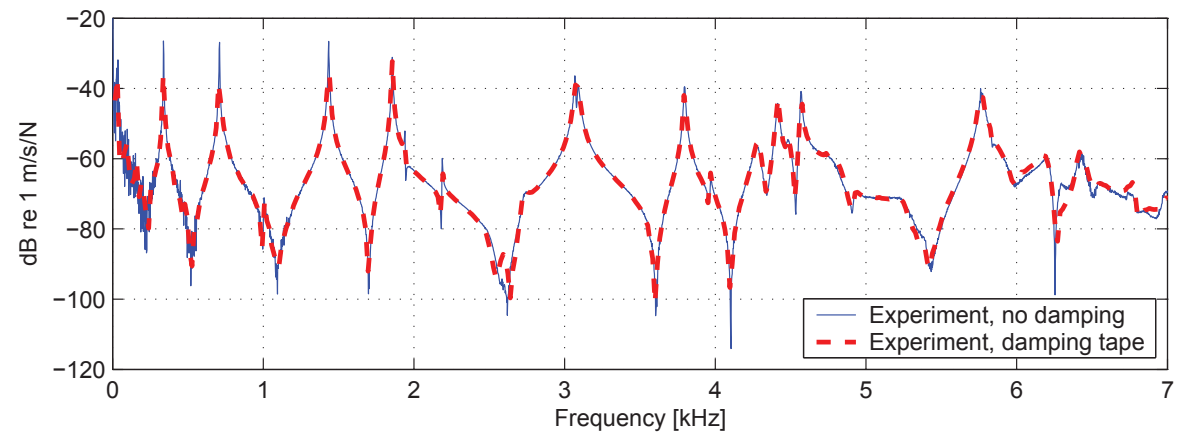

(b)

Fig. 9. Comparison of driving point mobilities for a simple plate, $(|\dot{w} / p|) \mathrm{dB}$ re $1 \mathrm{~m} / \mathrm{s} / \mathrm{N}$; (a) numerical calculations and (b) experimental measurements. Also shown are the mobilities when a single layer of damping tape is applied to the end of the plate. 


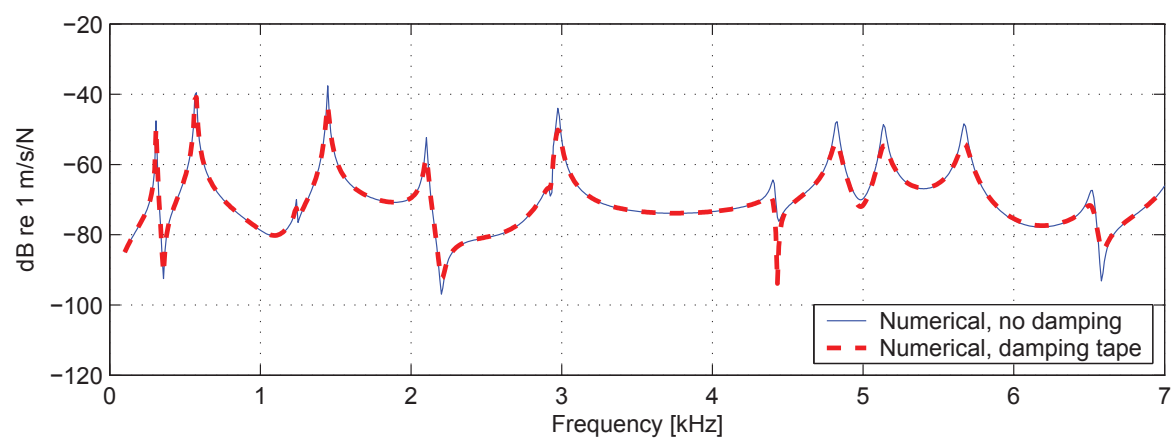

(a)

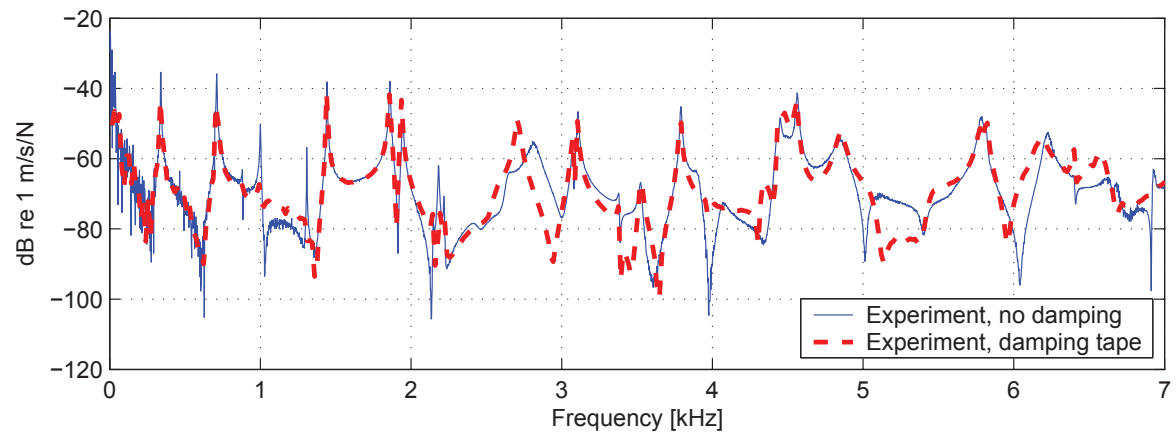

(b)

Fig. 10. Comparison of the cross point mobilities for a simple plate, $(|\dot{w} / p|) \mathrm{dB}$ re $1 \mathrm{~m} / \mathrm{s} / \mathrm{N}$; (a) numerical calculations and (b) experimental measurements. Also shown are the mobilities when a single layer of damping tape is applied to the end of the plate. 


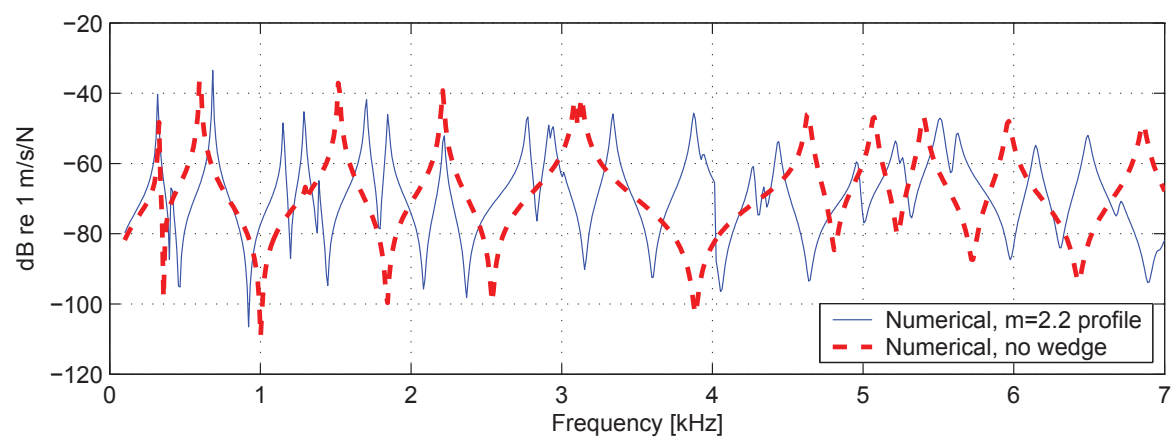

(a)

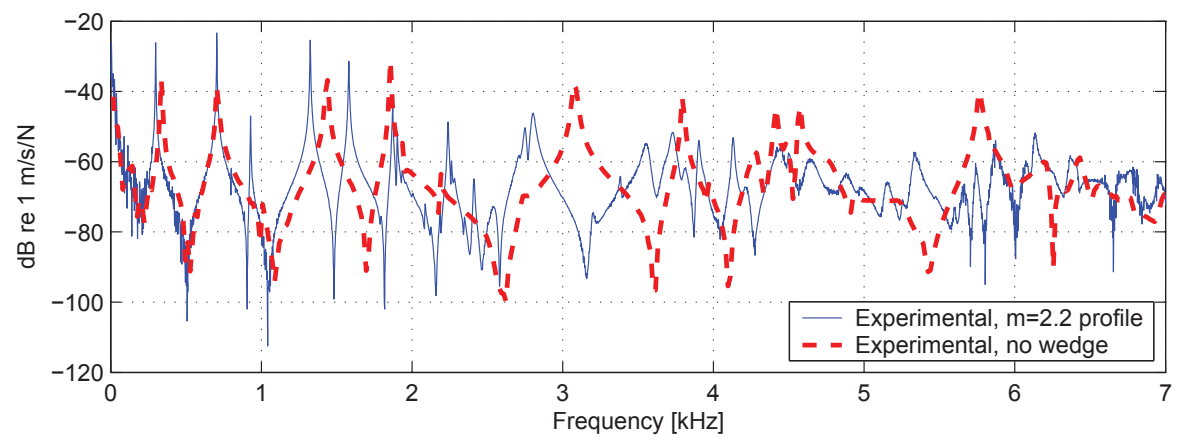

(b)

Fig. 11. Comparison of driving point mobilities, $(|\dot{w} / p|) \mathrm{dB}$ re $1 \mathrm{~m} / \mathrm{s} / \mathrm{N}$; (a) numerical calculations and (b) experimental measurements for a simple plate against a plate with a wedge shape using a $\mathrm{m}=2.2$ power-law profile. 


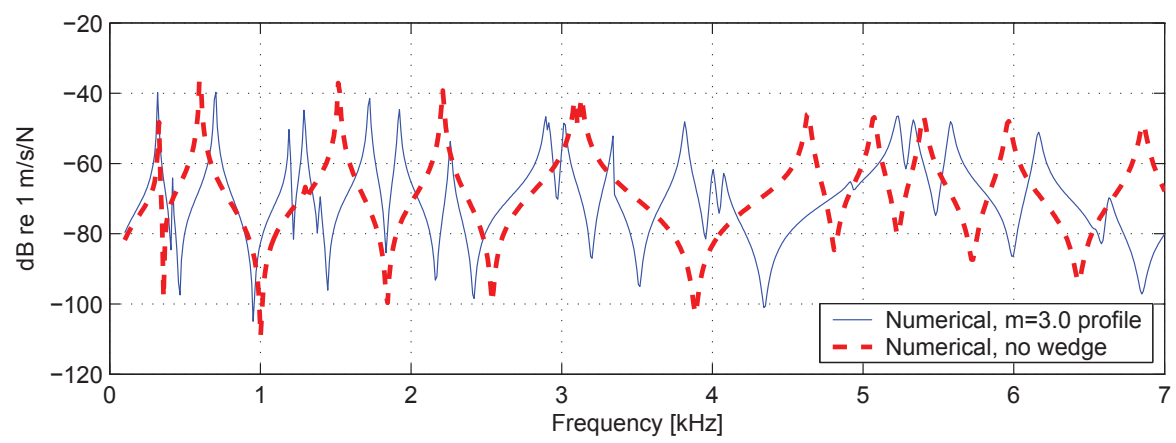

(a)

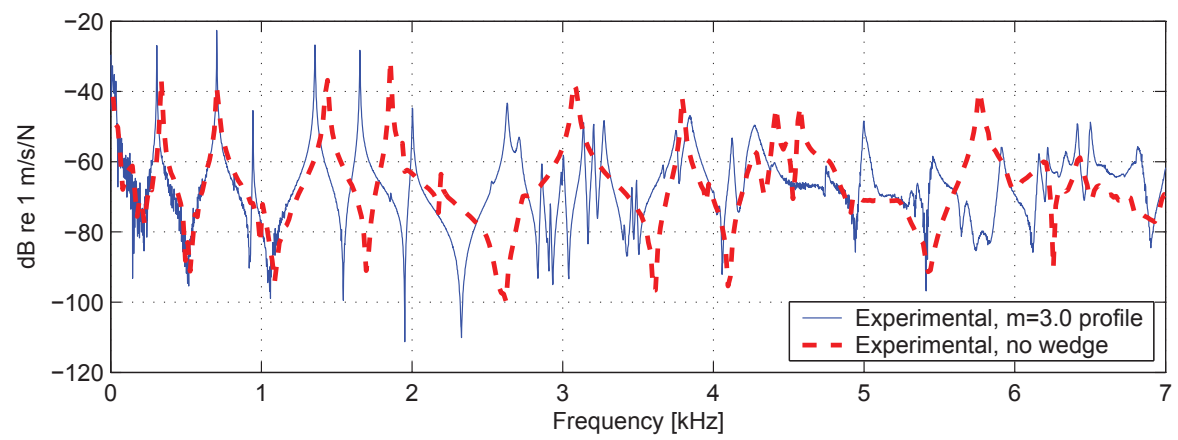

(b)

Fig. 12. Comparison of driving point mobilities for a simple plate against one with a profile of $\mathrm{m}=3.0$ power-law, $(|\dot{w} / p|) \mathrm{dB}$ re $1 \mathrm{~m} / \mathrm{s} / \mathrm{N}$; (a) numerical predictions and (b) experimental measurements. 


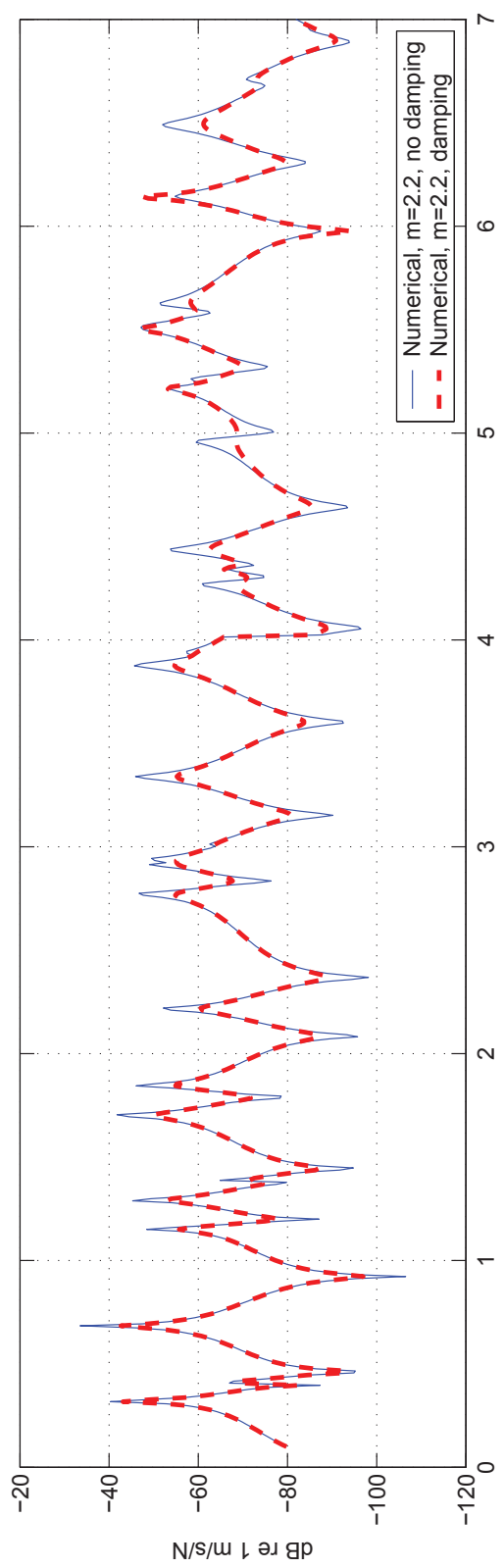

(a)

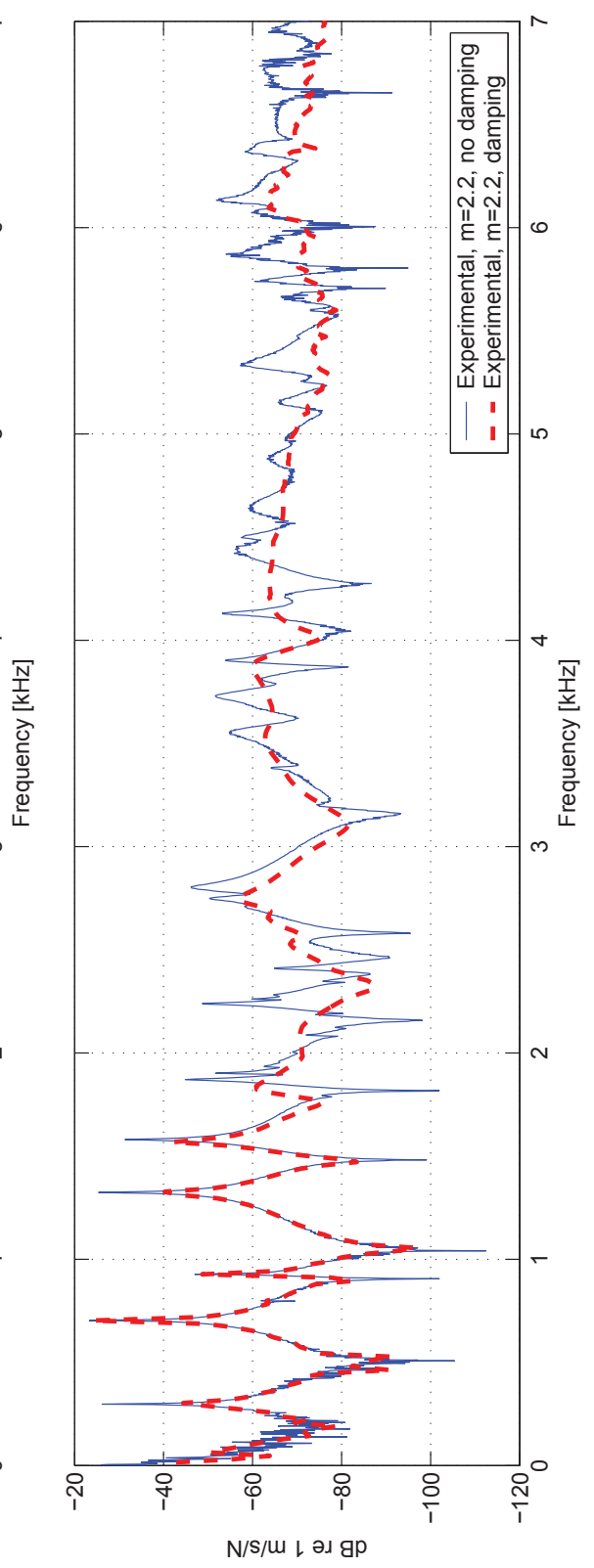

(b)

Fig. 13. Driving point mobilities for a plate with a wedge profile of power-law $\mathrm{m}=2.2$ without and with a single layer of damping tape applied; (a) numerical predictions and (b) experimental measurements. 


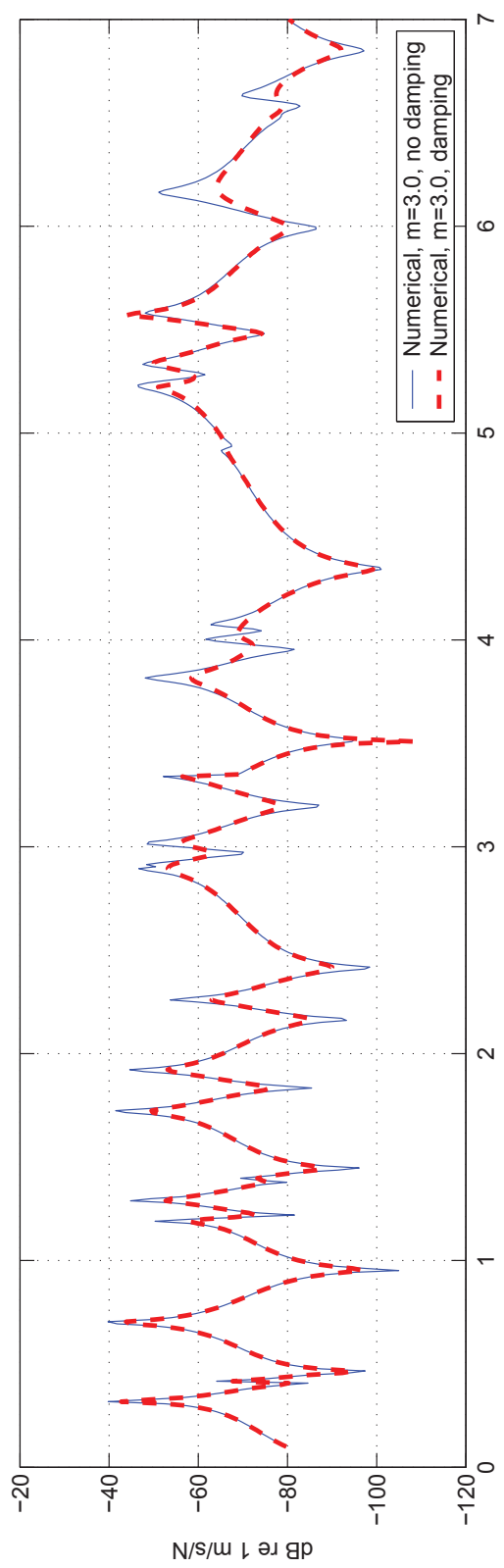

(a)

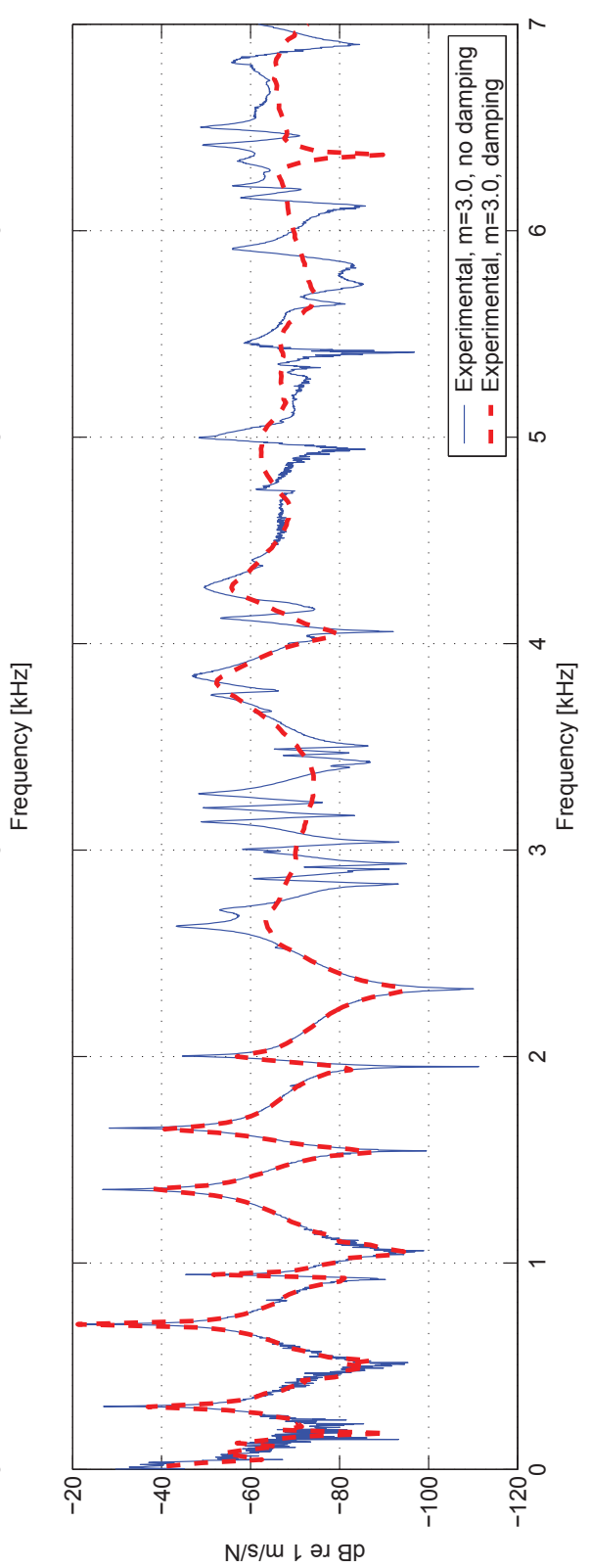

(b)

Fig. 14. Driving point mobilities for a plate with a wedge profile of power-law $m=3.0$ without and with a single layer of damping tape applied; (a) numerical predictions and (b) experimental measurements. 


\begin{tabular}{|c|c|c|c|}
\hline $\begin{array}{l}\qquad \text { Frequency range } / \mathrm{kHz} \\
\text { Average peak mobility in frequency } \\
\text { range (numerical predictions) }\end{array}$ & 1.0-3.0 & $3.0-5.0$ & $5.0-7.0$ \\
\hline Reference (plain plate) / dB & -39.0 & -46.5 & -48.0 \\
\hline $\begin{array}{l}\text { Reductions in average peak mobility } \\
\text { values compared to reference plate }\end{array}$ & & & \\
\hline Plain plate with damping film & -5.75 & -5.75 & -6.0 \\
\hline Plate with undamped wedge $(\mathrm{m}=2.2)$ & -8.5 & -6.0 & -3.75 \\
\hline Plate with damped wedge $(\mathrm{m}=2.2)$ & -18.0 & -13.0 & -5.0 \\
\hline Plate with undamped wedge $(\mathrm{m}=3.0)$ & -8.0 & -7.75 & -4.6 \\
\hline Plate with damped wedge $(\mathrm{m}=3.0)$ & -15.5 & -15.5 & -9.3 \\
\hline
\end{tabular}

Table 1

Summary of average peak point mobility measurements in a given frequency range $(\mathrm{dB})$, from the numerical predictions. The estimated reductions in $\mathrm{dB}$ are provided in reference to the plain rectangular plate in those same frequency bands.

\begin{tabular}{|l|c|c|c|}
\hline \multicolumn{1}{|c|}{ Frequency range / kHz } & $\mathbf{1 . 0 - 3 . 0}$ & $\mathbf{3 . 0 - 5 . 0}$ & $\mathbf{5 . 0 - 7 . 0}$ \\
$\begin{array}{l}\text { Average peak mobility in frequency } \\
\text { range (experimental measurements) }\end{array}$ & & & \\
\hline Reference (plain plate) / dB & -39.25 & -40.25 & -50.0 \\
\hline Reductions in average peak mobility & & & \\
values compared to reference plate & & & \\
Plain plate with damping film & -4.75 & -1.75 & -0.25 \\
Plate with undamped wedge $(\mathrm{m}=2.2)$ & 0.0 & -14.5 & -7.0 \\
Plate with damped wedge $(\mathrm{m}=2.2)$ & -10.75 & -23.5 & -18.25 \\
Plate with undamped wedge $(\mathrm{m}=3.0)$ & +3.5 & -8.5 & -5.0 \\
Plate with damped wedge $(\mathrm{m}=3.0)$ & -10.5 & -20.0 & -17.0 \\
\hline
\end{tabular}

Table 2

Summary of average peak point mobility measurements in a given frequency range $(\mathrm{dB})$, from the experimental measurements. The estimated reductions in $\mathrm{dB}$ are provided in reference to the plain rectangular plate in those same frequency bands. 